\title{
EFFECT OF GOVERNMENT SUBSIDIES ON SUPPLY CHAIN DECISION-MAKING AND COORDINATION IN THE CONTEXT OF COVID-19
}

\author{
SEn YANG AND WuYONG QIAN*
}

\begin{abstract}
Considering the two-stage supply chain composed of a leading retailer and a manufacturer under the background of covid-19 epidemic, the retailer determines the anti-epidemic effort level and bears the corresponding costs, and the manufacturer determines the cost sharing rate under the coordination strategy. This paper analyzes the pricing decision, anti-epidemic effort level and cost sharing rate of supply chain under different government subsidy measures and coordination strategies. From the perspective of Stackelberg game, we find that under the background of epidemic situation, government subsidies can improve the level of social welfare; the improvement of marginal income of anti-epidemic efforts is conducive to obtain a higher level of anti-epidemic efforts and social welfare; Within the reasonable marginal revenue range of anti-epidemic efforts, the coordination strategy can improve the retailers' anti-epidemic efforts and social welfare level, and the government can obtain the maximum anti-epidemic efforts and social welfare level by subsidizing manufacturers with cost sharing.
\end{abstract}

Mathematics Subject Classification. 90B99, 97M40.

Received October 21, 2020. Accepted June 1, 2021.

\section{INTRODUCTION}

Since the end of 2019, COVID-19 has ravaged the world, affecting hundreds of millions of human lives. In January 2020, Wuhan city, Hubei Province of the People's Republic of China first reported the novel Coronavirus pneumonia outbreak and took measures of "closing the cities" on January 23, 2020 to deal with the epidemic [34]. By 13 October 2020, more than 37 million people have been diagnosed with COVID-19 in almost all countries and regions of the world, and the cumulative number of deaths has reached more than 1.08 million [38]. When facing the epidemic situation, many countries in the world have developed obvious emergency and rescue measures. Cities with severe epidemic situation have taken measures of "closing the cities" to deal with the epidemic, major universities in the world have delayed the opening time of school, and many Internet enterprises allow employees to work at home, which shows the great impact of COVID-19. At the same time, the supply chain is also deeply affected.

COVID-19 makes communication between people more cautious. While some producers are busy, particularly in the food and healthcare sectors, they are a very small minority and most industries report a rapid deterioration in both demand and production. The disasters caused by epidemic outbreaks is different from other disasters, which will bring about severe disruptions in the supply chains and communities [18]. In severe

Keywords. COVID-19, government subsidy, supply chain management, coordination.

School of Business, Jiangnan University, 214122 Wuxi, P.R. China.

*Corresponding author: wuyongqian@jiangnan.edu.cn, qianyijiaemail@163.com

(C) The authors. Published by EDP Sciences, ROADEF, SMAI 2021

This is an Open Access article distributed under the terms of the Creative Commons Attribution License (https://creativecommons.org/licenses/by/4.0), which permits unrestricted use, distribution, and reproduction in any medium, provided the original work is properly cited. 
epidemic areas, manufacturers and retailers in the supply chain have increased production costs and normal production and processing activities are difficult to carry out due to problems such as difficult resumption of work and production, limited return of employees, difficult transportation of raw materials, blocked logistics transportation, slow capital turnover, and reduced social consumption capacity.

In fact, in such a harsh social environment, almost all supply chains would be less efficient if no measures were taken. In order to avoid social and economic imbalance, the government will subsidize the supply chain to improve the level of social production and economic environment. The government may provide a variety of subsidies, and there are various forms of subsidies, for example, green subsidies are provided to environment-friendly enterprises. Subsidies are a function of the green level of the supply chain to fit the concept of environmental protection and low-carbon. Government subsidies can be a function of total production, the Government of India Ministry of Power, through the UJALA - Unnat Jyoti By Affordable LEDs for All program, subsidized the procurement of LED light - bulbs in the country [33], this kind of subsidy is called unit production subsidy. In this paper, we will focus on cost subsidies and effort subsidies in order to better understand the diversity and complexity of subsidies.

However, even with government subsidies, the severe situation in the epidemic and the high cost of antiepidemic make the members of the supply chain face more and more challenges, because the additional costs are prohibitive for any company. Therefore, we analyzed the method of sharing the cost of anti-epidemic between retailers and manufacturers, that is, adopting appropriate coordination strategies to achieve the purpose of improving social welfare and supply chain efficiency. In the current situation, in the face of great difficulties, companies should unite and fight the epidemic together.

At present, it is not clear what is the optimal decision-making of the supply chain in determining the antiepidemic effort level, sales price, government subsidies and coordination strategy under the epidemic environment brought by COVID-19. What are the effects of different types of subsidies and coordination strategies on subsidy parameters, anti-epidemic efforts, supply chain performance and social welfare? To address the above issues, we summarize the literature relevant to our work in Section 2, present our model and calculate the optimal solution of the model in Section 3, and finally, discuss our findings and key insights in Sections 4 and 5 before giving our conclusions and suggestions for future research.

\section{Literature REVIEW}

Our study involves three literature streams as it addresses the impact of COVID-19 on the supply chain, social welfare and government policies, and coordination strategies in the supply chain.

Although the outbreak of novel coronavirus pneumonia was less than a year, the coronavirus pandemic is having a clear impact on the supply chains of virtually all manufacturers, retailers, and wholesalers [41]. The impact of COVID-19 on the supply chain is reflected in all aspects. Different types of supply chains are affected differently. Majumdar et al. studied the reasons behind the lack of social sustainability in the clothing supply chain in South Asian countries in the context of COVID-19 [31]. Rizou et al. summarized the possible transmission ways of COVID-19 through the food supply chain [39]. In addition, supply chain of critical onetime-use personal and protective equipment [40] and pharmaceutical supply chain [25] also indicate the wide impact of COVID19 on the supply chain. In addition, COVID-19 will not only affect a single supply chain, but also the supply chain network. Li et al. found that forward and backward disruption propagations became major stressors for supply chain network during the COVID-19 pandemic triggered by simultaneous and sequential supply and demand disruptions [28]. On the other hand, COVID-19 will pose challenges to sustainable supply chains, Karmaker et al. revealed the influential relationships and indispensable links between the drivers using fuzzy TISM to improve the SCS in the context of COVID-19 [24]. Specifically, the epidemic has reduced the face-to-face communication between people, thereby reducing the circulation of people [35]. For each member of the supply chain, this prevents employees from returning to their jobs normally. At the same time, the epidemic will lead to problems such as hindered logistics [36] and transportation and lowered socio-economic levels [29]. The various problems caused by COVID-19 will cause the supply chain to lose balance and vitality. Most of the 
papers in this field analyze the impact of COVID-19 from the overall supply chain, and our research will focus on the analysis of the decision-making of each subject in the supply chain in the context of COVID-19.

In the face of the severe COVID-19 epidemic situation, the government will do its part to make remedial measures [1,12], including emergency adjustments to the national budget and financial assistance to families in need, etc. Usually, the government will also subsidize the supply chain. Reviewing the literature, we find that the government's subsidy measures for the supply chain can be distinguished from the subsidy object, subsidy method, and supply chain type. Chen et al. have studied the optimal subsidy level of the government when the quantity of old products returned to the OEM and to consumers is uncertain, and found that no essential differences exist between providing subsidies directly to the manufacturer and to consumers [11]. Jiang et al. examined three possible subsidy provision strategies depending on which party (the power plant, the villagers' committee or the farmer) the government chooses to subsidise [23]. Wang et al. found that when the e-waste remanufacturing utilization rate is relatively high, the marginal effect of the subsidy to the remanufacturer on economic benefit and the recycle quantity increase as the subsidy increases, and the government should allocate as much support as possible to the remanufacturer [44]. From the way of subsidy, Jian et al. constructed the government cash subsidy strategy model based on the expected profits of supply chain members to analyze the government tax reduction subsidy strategy and cash subsidy strategy [22]. Han et al. found that government carbon subsidies are positive factors in the supply chain operation, which can stimulate the manufacturer to make low-carbon products as expected and choose the high quality-high price development mode [19]. Green consumption subsidy [32] and emission reduction subsidy [26] are also common forms of subsidies. The government will also subsidize different types of supply chains, Ma et al. studied the impact of consumption subsidies on the dual-channel closed-loop supply chain, and considered the impact of consumption subsidies from the perspectives of consumers, the size of the closed-loop supply chain and enterprises [30]. In order to cope with severe environmental problems and reduce the impact on shipping routes downstream of the ocean supply chain, Li et al. used game theory to determine the optimal government subsidy intensity and subsidy reduction point [27]. In the supply chain classification, green supply chain [43], low-carbon supply chain [45] and decentralized Reverse supply chain [20] are common. Social welfare is generally used as the objective function of government subsidies [5]. Our research specifically considers two different types of subsidies: manufacturer's production cost and retailer's anti-epidemic effort cost.

Our research is also closely related to the field of coordination strategy, appropriate coordination strategy can effectively improve supply chain performance [9]. Chen et al. found that using revenue sharing contracts, generate many more supply chain profiles for the PV supply chain comparing to the profiles eared by those supply chain strategies without any subsidy [8]. When Huang et al. studied the financing services of the third party logistics, he concluded that the profit of supply chain can achieve Pareto improvement, and the wholesale price contract may realise profit maximisation and channel coordination in the supply chain system under certain condition [21]. Ghosh et al. studied two models of cost sharing - one in which the retailer offers a cost sharing contract and the other in which the retailer and manufacturer bargain on the cost sharing contract [17]. In particular, we consider a special cost sharing strategy.

In summary, we establish a supply chain decision model in the context of pandemic, and analyze the influence of subsidy factors and coordination factors on optimal supply chain decision by comprehensively considering government subsidy factors and coordination mechanism. Combining mathematical analysis and example analysis, this paper investigates how to obtain the maximum effort level of anti-epidemic, the overall performance level of the supply chain and the level of social welfare, so as to provide some suggestions for the government and members of the supply chain to make decisions under the epidemic situation. This is not only conducive to the stability and sustainable development of the supply chain, but also contributes to the recovery of socio-economic status and the alleviation of the severity of the epidemic. 


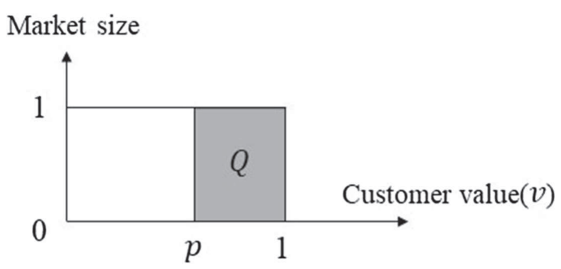

FiguRE 1. Distribution of customer value.

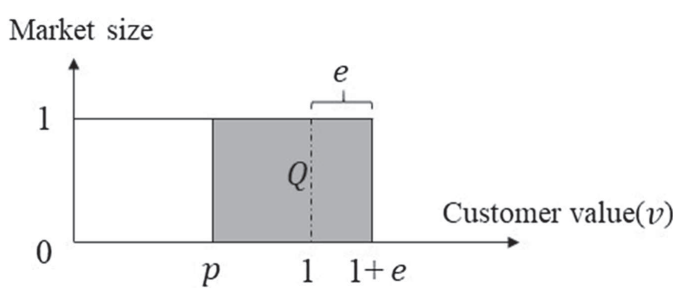

Figure 2. Distribution of customer value when considering $e$.

\section{THE MODEL}

\subsection{Model description}

We consider a secondary supply chain that is affected by the epidemic and is composed of upstream manufacturers $(M)$ and downstream retailers $(R)$. Manufacturers produce a specific type (not the products mentioned above that have increased sales during the epidemic). Compared with the normal period (no epidemic situation), manufacturers are in urgent need of local governments to implement subsidy measures due to the lack of human resources of employees, sales channels and the reduction of orders from downstream retailers.

On the other hand, under the impact of COVID-19, brick-and-mortar retailers in severely affected areas have been hit hard and do not have sufficient capacity to fight the epidemic. Therefore, this study mainly discusses online retailers that do not require high store rents (Hereinafter referred to as retailer). During the epidemic period, downstream retailers are responding to the local government's active anti-epidemic businessmen with social responsibility. Compared with the normal period, retailers need to bear greater risks when hiring employees, and the difficulty of logistics and transportation is greatly increased. The freight on the way will also increase with the severe social situation. We regard this retailer's behavior as a highly socially responsible anti-epidemic effort to compensate for the losses of manufacturers in severely affected areas. In order to obtain the marginal effect of the effort, suppose the retailer's anti-epidemic effort cost is quadratic type $\frac{\theta}{2} e^{2}$, where $e>0$ is the retailer's anti-epidemic effort level, and $\theta>0$ is the retailer's anti-epidemic effort cost coefficient, similar to Fan et al. [13], Atasu and Subramanian [3], Arora and Ceccagnoli [2], and Chen et al. [7]. Considering a product that is priced at $p$, the customer with a net surplus $v-p \geq 0$ will buy it [14]. From Figure 1, all the customers with valuations in the interval $[p, 1]$ will buy the product. Therefore, the demand of the product is $Q=\int_{p}^{1} \mathrm{~d} v=1-p$ for $0 \leq p \leq 1$. While making anti-epidemic efforts, retailers sell products to customers at the sales price $p$. Active and effective anti-epidemic efforts will reduce the severity of the epidemic and accelerate the progress of social recovery from the epidemic, which coincides with the will of consumers. Therefore, such anti-epidemic efforts meet the value needs of consumers. From Figure 2, all the customers with valuations in the interval $[p, 1+e]$ will buy the product. Therefore, the demand of the product is $Q=\int_{p}^{1+e} \mathrm{~d} v=1+e-p$ for $0 \leq p \leq 1$.

In order to accelerate the recovery of the economic level, the government will encourage all members of the supply chain to make efforts to control the epidemic, that is, the government will take subsidies for them [42], 


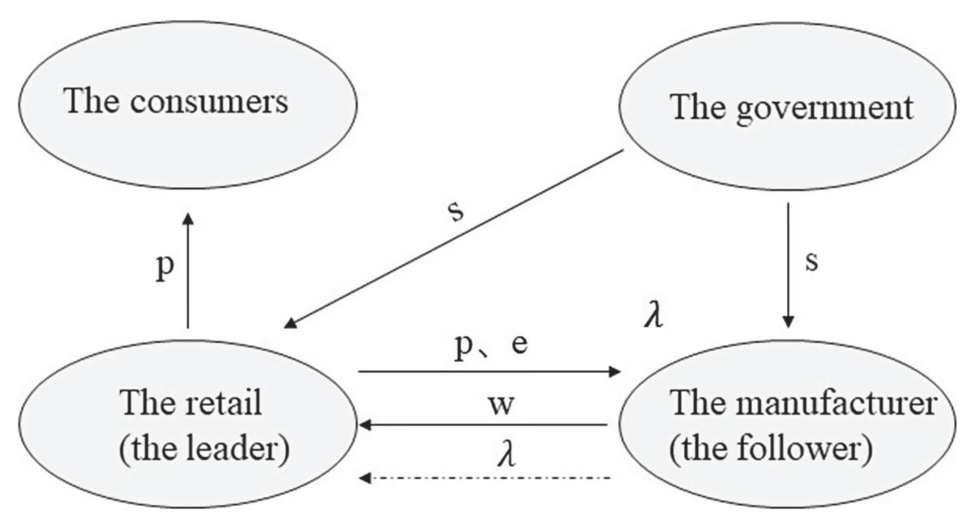

Figure 3. The decision system of supply chain.

and government subsidies are collectively referred to as $\Phi$. Considering a variety of subsidy methods, when subsidizing manufacturers, the government subsidizes the cost $c$ of each product produced by the manufacturer, then $\Phi=s Q$, where $s>0$ is the government's subsidy coefficient to the manufacturer, then the manufacturer's production cost per-unit of product is reduced to $c-s$. It should be noted that what we are talking about is the price and demand when the market clears. When subsidizing retailers, the government subsidizes the retailer's anti-epidemic effort level $e$, then $\Phi=s e$, where $s>0$ is the government subsidy coefficient to the retailer (the following describes how to distinguish the various subsidy coefficients), which means the greater the anti-epidemic efforts made by retailers, the more government subsidies they will receive. This is also one of the endogenous reasons for retailers' efforts to control the epidemic and develop the socio-economic level.

The government subsidy $s$ is determined by the government's profit function, that is, the social welfare function maximization [10]. Considering the increase in social benefits and the improvement effect of the epidemic EI brought about by the retailer's anti-epidemic efforts, government subsidies $\Phi$, total profits of the supply chain $\pi$, and consumer surplus GS as components of the social welfare function, so SW $=\mathrm{EI}-\Phi+\pi+$ GS. EI corresponds to the external benefits of retailers' continued anti-epidemic efforts. Different from the marginal benefit set by Raz et al. [37] and Atasu et al. [4], the unit of demand is a constant, i.e. $\mathrm{EI}=\alpha D$, and in order to reflect the continuous benefits of anti-epidemic efforts to social welfare, determine the best level of anti-epidemic efforts, and highlight the status of anti-epidemic efforts in the social welfare function, we define $\mathrm{EI}=\alpha e$, where $\alpha(0<\alpha<1)$ is the marginal benefit of the government's anti-epidemic efforts. The government subsidy $\Phi$ has been explained in the previous article, and $\Phi$ is different under different subsidy types. The third part is the total profit of the supply chain, that is, the sum of the profit of the manufacturer and the retailer. When calculating consumer surplus, we use $p_{0}$ to denote the price when demand is zero, i.e. $p_{0}=1+e$, then consumer surplus is denoted as $\left(p_{0}-p\right) D / 2$, and GS $=D^{2} / 2$ is calculated.

We analyze the influence of different government subsidy strategies on supply chain decision-making under the background of pneumonia caused by covid-19, considering three scenarios: the government subsidizes the manufacturer separately, the government subsidizes the retailer separately, and the government subsidizes both. Retailers' participation in the anti-epidemic activities will produce a lot of costs. In order to accelerate the progress of social recovery from the epidemic, ensure the stable operation of the supply chain and even improve the performance of the supply chain, manufacturers consider to take cost-sharing coordination measures with retailers. Similar to Cao et al. [5] and Fan et al. [13], we study the situation that manufacturers and retailers share the cost of anti-epidemic efforts of retailers. In particular, it is assumed that the proportion of antiepidemic effort cost borne by retailers is $\lambda$, and the remaining $1-\lambda$ is borne by manufacturers. The order of the game can be expressed as Figure 3:

The following table summarizes all the symbols that appear in this section: 
Summary of main notations

\begin{tabular}{ll}
\hline Term & Meaning \\
\hline$c$ & Manufacturer's unit cost \\
$\omega$ & Wholesale price \\
$p$ & Retail price \\
$\theta$ & Retailers' anti-epidemic effort cost coefficient \\
$e$ & Retailers' anti-epidemic effort level \\
$D$ & Demand \\
$s$ & Government subsidy coefficient \\
$\Phi$ & Government subsidies \\
$\alpha$ & The marginal benefit of the government's anti-epidemic efforts \\
$\lambda$ & The proportion of anti-epidemic efforts undertaken by retailers \\
EI & Increased social benefits and improved epidemic situation \\
GS & Consumer surplus \\
$\pi$ & Profit \\
SW & Social welfare \\
\hline
\end{tabular}

\subsection{Model calculation}

For ease of exposition, we define System $x y$, where $x \in\{n, c, e, b\}$ stands for the government does not subsidize, the government subsidizes the manufacturer's cost of the product, the government subsidizes the retailer's level of effort and government subsidizes both, and $y \in\{Y, N\}$ represents whether or not to adopt a coordination strategy. For example, System $e N$ indicates that the government subsidizes the effort of retailers and those manufacturers and retailers do not adopt a coordinated strategy.

\subsubsection{System $n N$}

In the case of System $n N$, the government does not subsidize members of the supply chain, i.e. $\Phi=0$, and the manufacturer and retailer do not adopt coordination strategy, i.e. $\lambda=1$. In this case, the overall benefits of the manufacturer, retailer and supply chain are:

$$
\begin{aligned}
\pi^{M} & =(\omega-c) q \\
\pi^{R} & =(p-\omega) q-\frac{\theta}{2} e^{2} \\
\pi^{V} & =\pi^{M}+\pi^{R} .
\end{aligned}
$$

Similarly, we can derive the expression of supply chain members and social welfare in the remaining cases.

\subsubsection{System $n Y$}

$$
\begin{aligned}
\pi^{M} & =(\omega-c) q-\frac{\theta(1-\lambda)}{2} e^{2} \\
\pi^{R} & =(p-\omega) q-\frac{\theta \lambda}{2} e^{2} \\
\pi^{V} & =\pi^{M}+\pi^{R} .
\end{aligned}
$$

\subsubsection{System $c N$}

$$
\begin{aligned}
\pi^{M} & =\left(\omega-c+s_{c N}\right) q \\
\pi^{R} & =(p-\omega) q-\frac{\theta}{2} e^{2}
\end{aligned}
$$




$$
\mathrm{SW}=\alpha e-s_{c N} q+\pi^{M}+\pi^{R}+\frac{q^{2}}{2}
$$

\subsubsection{System $c Y$}

$$
\begin{aligned}
\pi^{M} & =\left(\omega-c+s_{c Y}\right) q-\frac{\theta(1-\lambda)}{2} e^{2} \\
\pi^{R} & =(p-\omega) q-\frac{\theta \lambda}{2} e^{2} \\
\mathrm{SW} & =\alpha e-s_{c Y} q+\pi^{M}+\pi^{R}+\frac{q^{2}}{2} .
\end{aligned}
$$

\subsubsection{System eN}

$$
\begin{aligned}
\pi^{M} & =(\omega-c) q \\
\pi^{R} & =(p-\omega) q-\frac{\theta}{2} e^{2}+s_{e N} e \\
\mathrm{SW} & =\alpha e-s_{e N} q+\pi^{M}+\pi^{R}+\frac{q^{2}}{2} .
\end{aligned}
$$

\subsubsection{System $e Y$}

$$
\begin{aligned}
\pi^{M} & =(\omega-c) q-\frac{\theta(1-\lambda)}{2} e^{2} \\
\pi^{R} & =(p-\omega) q-\frac{\theta \lambda}{2} e^{2}+s_{e Y} e \\
\mathrm{SW} & =\alpha e-s_{e Y} q+\pi^{M}+\pi^{R}+\frac{q^{2}}{2} .
\end{aligned}
$$

\subsubsection{System $b N$}

$$
\begin{aligned}
\pi^{M} & =\left(\omega-c+s_{b N}\right) q \\
\pi^{R} & =(p-\omega) q-\frac{\theta}{2} e^{2}+s_{b N} e \\
\mathrm{SW} & =\alpha e-s_{b N} q+\pi^{M}+\pi^{R}+\frac{q^{2}}{2} .
\end{aligned}
$$

In accordance with the game sequence given in the previous article, we use reverse induction to solve the supply chain decision. In order to avoid the length of the text, we show the specific equilibrium solution solving process in Appendix A. While ensuring the positive constraints of the parameters and the second-order optimal conditions, we will further analyze and discuss the equilibrium solution.

\section{Managerial implications}

In this section, we first analyze the endogenous causes of government subsidies in the context of epidemic situation when the national finance is in crisis. Section 4.1 compares the social welfare level with or without government subsidies and the optimal retailer's anti-epidemic effort level. Then we compared some equilibrium results for all five subsidies $c N, c Y, e N, e Y$ and $b N$. Sections 4.1 and 4.2, respectively analyzed and calculated the best retailers' anti-epidemic effort level and the best social profit level. Then, considering the impact of the cost coefficient, we discussed the impact of the retailer's anti-epidemic effort cost coefficient on some equilibrium results in various situations, this analysis is shown in Section 4.3. In order to facilitate the analysis, this article takes $\alpha=0.1$ and $1<\theta<3$ (satisfy the quadratic conditions in the appendix) for analysis. For the main idea of not overcrowding, our interested readers refer to Appendix B, which contains all the calculations and proofs in this section. 


\subsection{Supply chain decision analysis with or without government subsidy}

In this section, we compare and analyze the optimal decision of supply chain with or without government subsidy. To be specific, Proposition 4.1a compared and analyzed the optimal level of anti-epidemic efforts with and without government subsidies, while Proposition 4.1b examined the level of social welfare with and without government subsidies.

Summarily, we write the result as:

Proposition 4.1. (a) $e_{c N}>e_{n N}, e_{e N}>e_{n N}, e_{b N}>e_{n N}, e_{c Y}>e_{n Y}, e_{e Y}>e_{n Y}$. (b) $\mathrm{SW}_{c N}>\mathrm{SW}_{n N}, \mathrm{SW}_{e N}>\mathrm{SW}_{n N}, \mathrm{SW}_{b N}>\mathrm{SW}_{n N}, \mathrm{SW}_{c Y}>\mathrm{SW}_{n Y}, \mathrm{SW}_{e Y}>\mathrm{SW}_{n Y}$.

Proposition 4.1a shows that, regardless of whether coordination strategy is considered, the optimal level of anti-epidemic efforts is always higher with government subsidies than without government subsidies. Obviously, when the government subsidizes the retailers, the increase of income brought by the improvement of antiepidemic efforts is greater than the increase of cost, and the government subsidies have a positive impact on the "anti-epidemic efforts". At the same time, with the improvement of anti-epidemic efforts, the overall performance of the supply chain, consumer surplus and marginal income of anti-epidemic efforts will also increase, so will the level of social welfare. When the government subsidizes the manufacturer, the unit cost of the manufacturer will be reduced, the retailer can get a lower wholesale price in the process of bargaining with the manufacturer, more cost will be used to improve the level of anti-epidemic efforts, the overall performance of the supply chain, consumer surplus and the sustainable income of anti-epidemic efforts will also be increased, so the level of social welfare will also be improved.

\subsection{Comparison of retailers' anti-epidemic efforts and coordination levels}

In this section, we examine the impact of various subsidy measures on the level of anti-epidemic efforts. Specifically, Proposition 4.2a examined the optimal anti-epidemic effort level in several situations without a coordinated strategy; Proposition 4.2b compared the optimal anti-epidemic effort level when a coordinated strategy is adopted and when a coordinated strategy is not adopted; Proposition 4.2c compared the coordination levels under the two coordination strategies.

Summarily, we write the result as:

Proposition 4.2. Comparison of the level of anti-epidemic efforts and coordination levels under different subsidies

(a) $e_{c N}<e_{e N} \Leftrightarrow \psi_{e 1}>0 ; e_{c N}<e_{b N} \Leftrightarrow \psi_{e 2}>0 ; e_{e N}<e_{b N}$;

(b) $e_{e N}<e_{e Y}<e_{c Y} ; e_{c N}<e_{c Y} \Leftrightarrow \psi_{e 3}<0$;

(c) $\lambda_{c Y}<\lambda_{e Y}$

where $\psi_{e 1}$ and $\psi_{e 2}$ are defined in the proof.

Proposition $4.2 \mathrm{a}$ analyzes the optimal $e$ without considering the coordination strategy. When the government subsidizes $e$ and $c$ at the same time, the optimal level of anti-epidemic effort is always greater than that when the government only subsidizes $e$. This shows that under the premise of subsidizing $e$, when the government subsidizes $c$ again, the unit cost of the manufacturer will be reduced, the retailer can get a lower wholesale price in the process of bargaining with the manufacturer, more costs will be used to improve the anti-epidemic effort level, and the optimal anti-epidemic effort level will be improved accordingly. Figure 4 shows that when $\psi_{e 1}>0$ is satisfied, the optimal level of anti-epidemic effort when the government subsidizes $e$ is greater than that when the government subsidizes $c$, this shows that when the government subsidizes $e$, the increase of subsidies has a greater incentive effect on retailers' efforts to improve the anti-epidemic level than when the government subsidizes $c$, the decrease of wholesale price has a greater incentive effect on retailers' efforts to improve the anti-epidemic level. Figure 5 shows that when $\psi_{e 2}>0$ is satisfied, the government provides subsidies to $e$ and $c$ at the same time, the optimal anti-epidemic effort level is larger than that of the government subsidies alone. 


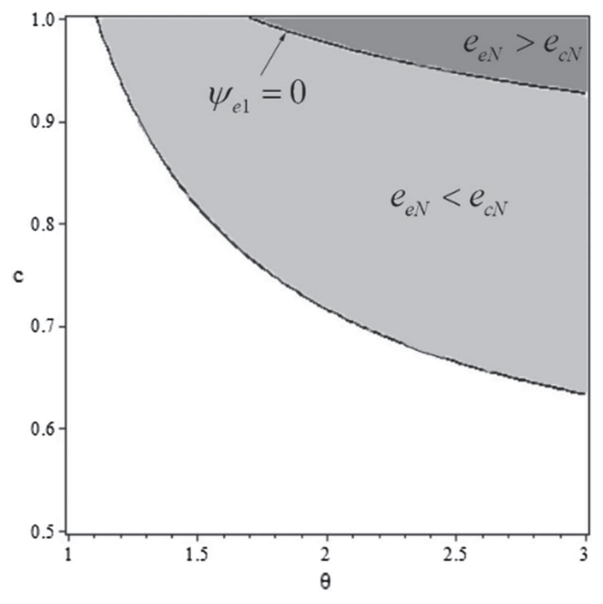

Figure 4. Comparison of $e_{e N}$ and $e_{c N}$.

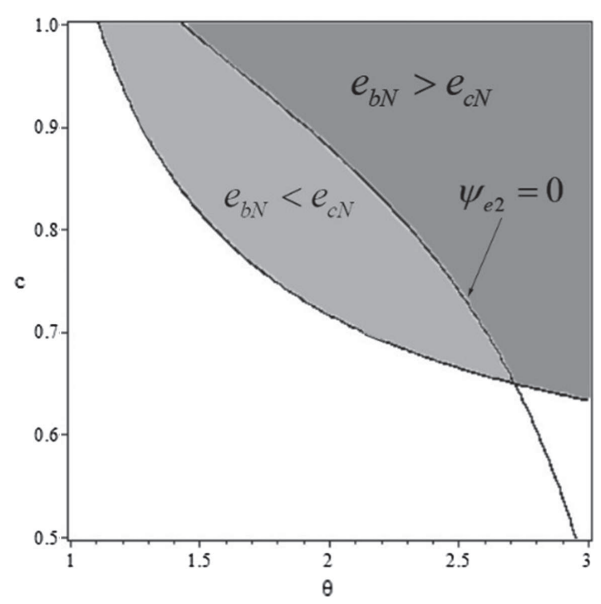

Figure 5. Comparison of $e_{c N}$ and $e_{b N}$.

In other words, when $\psi_{e 2}>0$, on the premise of subsidizing $c$, the government subsidizes $e$ again, the cost increase caused by improving the level of anti-epidemic efforts is less than the subsidy amount, so the level of anti-epidemic efforts is increased.

Proposition $4.2 \mathrm{~b}$ shows that coordination strategy can improve the optimal level of anti-epidemic efforts in supply chain when government subsidies $e$. A coordinated strategy means that manufacturers bear part of the cost of anti-epidemic efforts, while retailers have a lower cost of anti-epidemic efforts, which can motivate retailers to increase the level of anti-epidemic efforts. Figure 6 shows that in the case of government subsidy $c$, the coordination strategy can improve the optimal anti-epidemic effort level of the supply chain only when $\psi_{e 3}>0$ is satisfied. It can be found from the observation in Figure 6 that, within a reasonable range, the possibility of $\psi_{e 3}>0$ (i.e. $e_{c N}<e_{c Y}$ ) is high, which indicates that when manufacturers receive government subsidies, they have a strong willingness to share the anti-epidemic effort with retailers, this is because when the government subsidizes $c$, the manufacturer's unit cost is reduced, and more of the cost can be used to share the cost of anti-epidemic efforts. Finally, we conclude from $e_{e Y}<e_{c Y}$ that when the government subsidizes $c$, the 


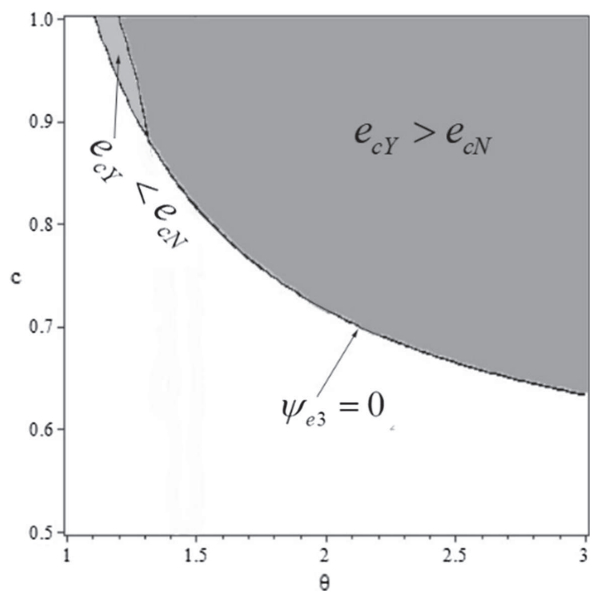

FIGURE 6. Comparison of $e_{c N}$ and $e_{c Y}$.

coordination strategy adopted by the supply chain subjects has a more stimulating effect on the improvement of anti-epidemic efforts.

Proposition 4.2c shows that the coordination level of System $e Y$ is always higher than that of System $c Y$, because when the government subsidizes $c$, manufacturers are more willing to share the cost of anti-epidemic efforts.

\subsection{Comparison of social welfare levels}

In this section, we examine the effects of various subsidy measures on the level of social welfare. To be specific, Proposition 4.3a compares the optimal level of social welfare in several situations without coordination strategy, while Proposition 4.2b compares the optimal level of social welfare in the case of coordination strategy and without coordination strategy.

Summarily, we write the result as:

Proposition 4.3. Comparison of social welfare levels under different subsidy types.

(a) $\mathrm{SW}_{b N}<\mathrm{SW}_{e N}<\mathrm{SW}_{c N}$;

(b) $\mathrm{SW}_{e Y}<\mathrm{SW}_{c Y} ; \mathrm{SW}_{e N}<\mathrm{SW}_{e Y} \Leftrightarrow \psi_{\mathrm{SW} 1}>0 ; \mathrm{SW}_{c N}<\mathrm{SW}_{c Y} \Leftrightarrow \psi_{\mathrm{SW} 2}>0$;

where $\psi_{\mathrm{SW} 1}$ and $\psi_{\mathrm{SW} 2}$ are defined in the proof.

Proposition 4.3a shows that, without considering the coordination strategy, for any given type of subsidies, the social welfare function presents an absolute relationship, that is, the social welfare function is the largest when the government subsidizes $c$, followed by the government subsidizes both $c$ and $e$, and the smallest is the government subsidizes $e$. This shows that when the government subsidizes the manufacturer, the manufacturer's unit cost will be reduced, and the retailer can get lower wholesale price in the process of bargaining with the manufacturer. More cost of the retailer will be used to improve the anti-epidemic effort level, and then the optimal anti-epidemic effort level will be increased. Then the marginal benefits of anti-epidemic efforts, overall benefits of the supply chain and consumer surplus all increase, and the increase rate is higher than the increase rate of government subsidies (the difference between the two changes is set as $\Delta \varsigma_{c N}$ ), so the level of social welfare increases. Similarly, $\Delta \varsigma_{c N}>\Delta \varsigma_{b N}>\Delta \varsigma_{e N}$ can be obtained from Proposition 4.3a.

Proposition $4.3 \mathrm{~b}$ shows that when $\psi_{\mathrm{SW} 2}>0$ is established in Figure 7 , the coordination strategy can improve the optimal social welfare level of the supply chain when $c$ is subsidized by the government. Adopting coordination strategy means that manufacturers bear part of the cost of anti-epidemic efforts, and retailers' cost 


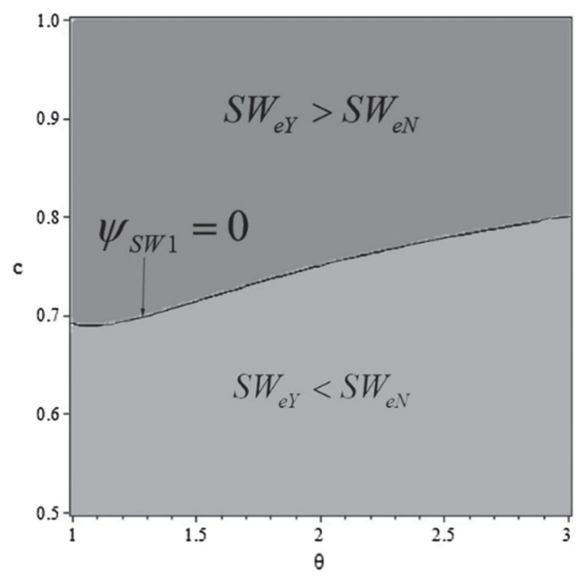

Figure 7. Comparison of $\mathrm{SW}_{e N}$ and $\mathrm{SW}_{e Y}$.

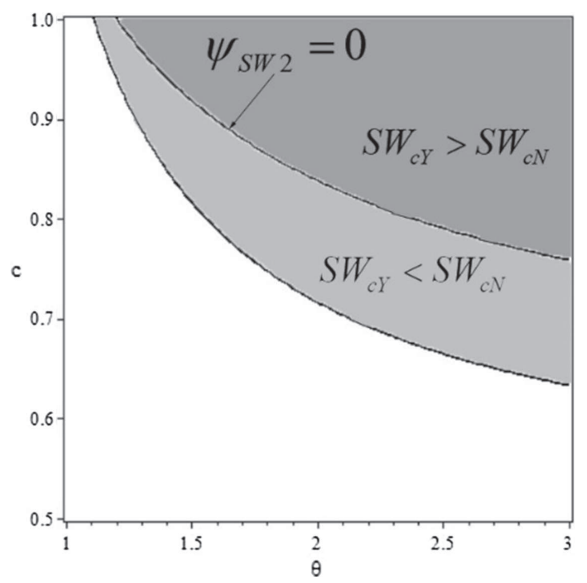

Figure 8. Comparison of $\mathrm{SW}_{c N}$ and $\mathrm{SW}_{c Y}$.

of anti-epidemic efforts is reduced, which can encourage retailers to improve the level of anti-epidemic efforts. With the improvement of anti-epidemic efforts, the cost of government subsidies increases, and the increase rate is higher than the marginal benefit of anti-epidemic efforts, the overall benefit of supply chain and the increase rate of consumer surplus. Therefore, when the government subsidies $c$, the coordination strategy will reduce the level of social welfare. Similarly, we get $\Delta \varsigma_{c Y}>\Delta \varsigma_{e Y}$. When analyzing System $e N$ and System $e Y$, we find that they are similar to $e_{c N}$ and $e_{c Y}$ in Section 4.2. As shown in Figure 8, the optimal social welfare function presents different relationships under different constraints. When $\psi_{\mathrm{SW} 1}>0$, there is $\Delta \varsigma_{e Y}>\Delta \varsigma_{e N}$, that is, coordination strategy can improve the optimal level of social welfare when the government subsidizes $e$.

\subsection{The impact of retailer's anti-epidemic effort cost coefficient}

\section{Proposition 4.4.}

$$
\frac{\partial e_{c N}}{\partial \theta}<0, \frac{\partial e_{c Y}}{\partial \theta}<0, \frac{\partial e_{e N}}{\partial \theta}<0, \frac{\partial e_{e Y}}{\partial \theta}<0, \quad \text { and } \quad \frac{\partial e_{b N}}{\partial \theta}<0
$$




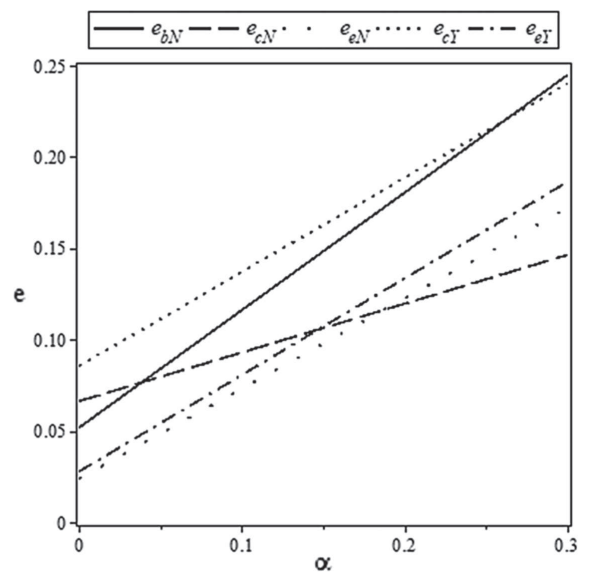

FiguRE 9. The relationship between $e$ and $\alpha$.

\section{Proposition 4.5.}

$$
\frac{\partial \mathrm{SW}_{c N}}{\partial \theta}<0, \frac{\partial \mathrm{SW}_{c Y}}{\partial \theta}<0, \frac{\partial \mathrm{SW}_{e N}}{\partial \theta}<0, \frac{\partial \mathrm{SW}_{e Y}}{\partial \theta}<0, \quad \text { and } \quad \frac{\partial \mathrm{SW}_{b N}}{\partial \theta}<0 .
$$

Proposition 4.4 shows that when the cost coefficient $\theta$ of anti-epidemic efforts of retailers increases, the optimal anti-epidemic effort level under five kinds of subsidies will decrease. Obviously, when $\theta$ increases, retailers will incur more costs if they take the same actions to fight the epidemic, which will discourage retailers' enthusiasm for fighting the epidemic, and the optimal level of fighting the epidemic will decrease accordingly.

Proposition 4.5 shows that when the cost coefficient $\theta$ of anti-epidemic efforts of retailers increases, the optimal level of social welfare under five kinds of subsidies will decrease. Obviously, the expansion of $\theta$ will reduce the overall benefit of the supply chain, the marginal income of the anti-epidemic efforts will also decrease, and the level of social welfare will also decline.

\section{NumericAl ANALYSIS}

The above model mainly analyzes the optimal decision when the government has different subsidy strategies. Next, we assign the relevant parameters in the model to further analyze and verify the relevant conclusions. From the above analysis, it can be seen that the marginal benefit $\alpha$ of the government's anti-epidemic efforts is the main variable affecting the decision-making of the supply chain. Therefore, we select $c=0.9, \theta=2.5$ within an appropriate range to focus on the analysis of the impact of the marginal benefit of the anti-epidemic efforts.

The relationship between the level of anti-epidemic efforts and the marginal benefits of anti-epidemic efforts is shown in Figure 9. As can be seen from Figure 9, $e$ is in a positive linear relationship with $\alpha$. When the marginal benefit of anti-epidemic efforts increases, the optimal anti-epidemic effort level of retailers will also increase. When $\alpha=0.1$, the anti-epidemic effort level in System $c Y$ is the highest, which verifies Proposition 4.2. At the same time, we also found that no matter which entity is subsidized separately by the government, the cost-sharing strategy is always conducive to improving the level of anti-epidemic efforts of retailers. When the marginal benefit of anti-epidemic effort is within the normal level $(\alpha \in(0,0.262))$, System $c Y$ is always the best choice, because the optimal anti-epidemic effort level is the highest at this time.

Figure 10 shows that the profit of manufacturers increases with the increase of the marginal benefit of antiepidemic efforts. When the government subsidizes $e$, adopting a coordinated strategy means that manufacturers share the cost of anti-epidemic efforts of retailers. At this time, the cost of anti-epidemic efforts of retailers will decrease, and retailers will increase the level of anti-epidemic efforts, and the demand for products will 


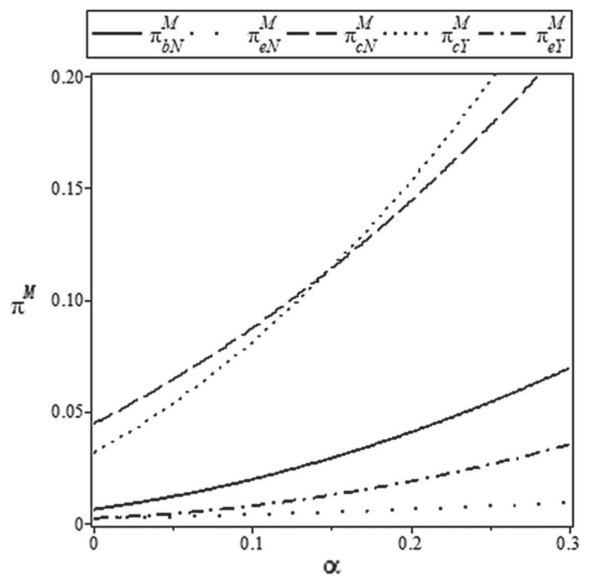

FIGURE 10. The relationship between $e$ and $\pi^{M}$.

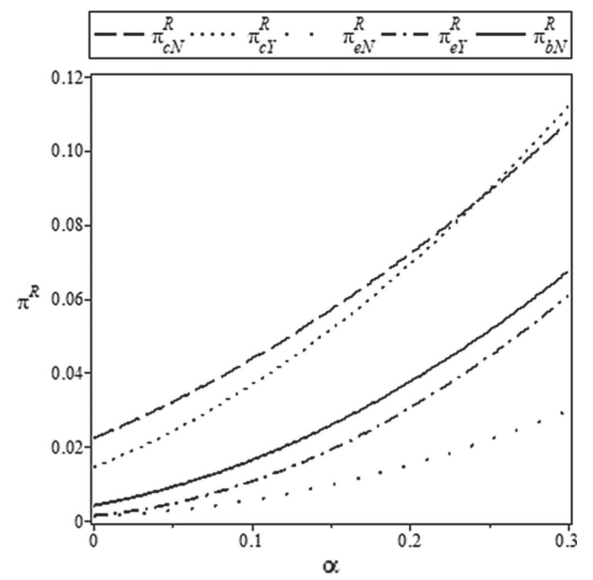

FIGURE 11. The relationship between $e$ and $\pi^{R}$.

increase accordingly. The increase in orders has increased profits by more than the share of the cost of the anti-epidemic effort, so manufacturers' profits have increased. For manufacturers, when the marginal benefit of anti-epidemic efforts is low $(\alpha \in(0,0.147))$, the manufacturer's profit under System $c N$ is the highest; when the marginal benefit of anti-epidemic efforts is high $(\alpha \in(0.147,0.3))$, the manufacturer's profit under System $c Y$ is the highest.

Figure 11 shows that the retailer's profit increases with the increase of the marginal benefit of anti-epidemic efforts. When the government subsidizes $e$, adopting a coordinated strategy means that manufacturers share the cost of anti-epidemic efforts of retailers. At this time, the cost of anti-epidemic efforts of retailers decreases, retailers will increase the level of anti-epidemic efforts, and the demand for products will increase accordingly. Higher orders and higher government subsidies have increased profits more than the cost of the anti-epidemic effort, so retailers' profits have increased. For retailers, when the marginal benefit of anti-epidemic efforts is at a normal level $(\alpha \in(0,0.244))$, the manufacturer's profit under System $c N$ is the highest; when the marginal benefit of anti-epidemic efforts is higher $(\alpha \in(0.244,0.3))$, the manufacturer's profit under System $c Y$ is the highest. 


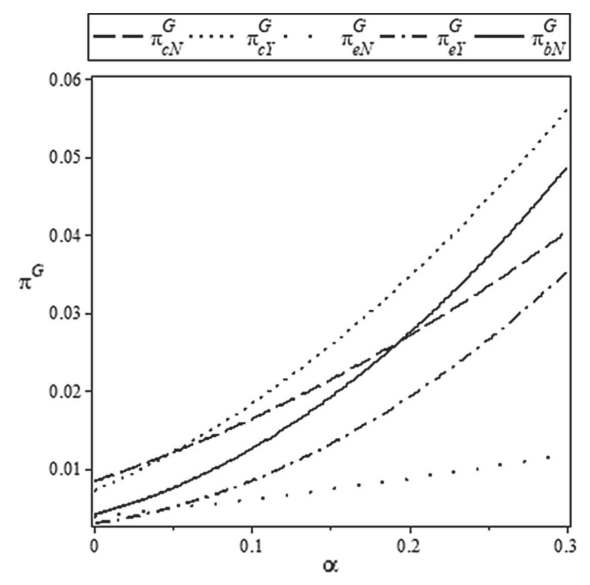

FiguRE 12. The relationship between $e$ and $\pi^{G}$.

The relationship between the level of social welfare and the marginal benefits of anti-epidemic efforts is shown in Figure 12. As can be seen from Figure 12, SW is positively correlated with SW. When the marginal benefit of anti-epidemic efforts increases, the level of social welfare will also increase. When $\alpha=0.1$, the social welfare level in the case of System $c Y$ is the highest, which verifies Proposition 4.3. At the same time, we also find that no matter which subject is subsidized by the government alone, when the marginal benefit of anti-epidemic efforts is not within the minimum range $(\alpha \in(0.048,0.3))$, the cost-sharing strategy is always conducive to improving the anti-epidemic efforts of retailers, and the System $c Y$ is always the optimal choice, because the social welfare level is the highest at this time.

\section{MANAGERIAL INSIGHTS AND FUtURE RESEARCH DIRECTIONS}

\subsection{Managerial insights}

In the context of COVID-19, enterprises in the supply chain actively participate in the fight against the epidemic and may share the additional costs arising from the fight against the epidemic, and the government will provide cost subsidies and anti-epidemic efforts subsidies to enterprises in the supply chain. We build a model to analyze the operation of the supply chain, the optimal solution of the supply chain when the social welfare is maximized, and the influence of relevant parameters on the optimal solution. The analysis results are summarized as follows.

In the context of the pandemic, the social and economic benefits decrease and the government will face a certain financial crisis. However, when the government takes appropriate subsidy measures for manufacturers and retailers, the benefits of members of the supply chain, the overall performance level of the supply chain and the level of social welfare will increase. Therefore, the government has an incentive to subsidize members of the supply chain, which can improve socio-economic benefits in the context of the epidemic, contribute to socio-economic recovery, and reduce the socio-economic impact of the epidemic. On the other hand, it is obvious that with the increase of the marginal benefits of the anti-epidemic efforts, the better the effect of government subsidies on the improvement of social welfare level will be. Generally speaking, the measures taken by the government to improve the marginal benefits of anti-epidemic efforts include: actively publicizing the anti-epidemic efforts of members of the supply chain to mobilize consumers' purchase desire; We will actively introduce epidemic prevention and control policies, improve the efficiency of enterprises' resumption of work and production, speed up the efficiency of the circulation of materials and products, and improve the logistics efficiency in the supply chain. Accelerating the breakthrough of technical barriers and the research and development of key logistics 
technologies are conducive to improving the efficiency of enterprises' efforts to fight against the epidemic, so as to improve the marginal income of efforts to fight against the epidemic.

The government has the motivation to subsidize the members of the supply chain. Under the marginal benefits of different anti-epidemic efforts, different subsidy measures can maximize the anti-epidemic effort level or social welfare level. When the coordination strategy is not taken into account, the social welfare will be the largest when the government chooses to subsidize the manufacturer $(\alpha \in(0,0.194))$, and the social welfare will be the largest when the marginal benefit of the anti-epidemic efforts exceeds this range $(\alpha \in(0.194,0.3))$. When we analyze the optimal decision of supply chain, we find that the cost sharing strategy can effectively improve the anti-epidemic effort level of retailers and social welfare level.

By comprehensively considering all subsidy scenarios, it can be concluded that when the marginal benefits of anti-epidemic efforts are within the normal range $(\alpha \in(0.048,0.242))$, and when the government subsidizes manufacturers in the supply chain that adopt the cost-sharing strategy, the anti-epidemic efforts and social welfare levels are superior to those of other subsidy strategies. Therefore, the following implications can be drawn. Under the epidemic background, member enterprises in the supply chain should actively adopt the costsharing strategy of anti-epidemic efforts, which can improve the profit level of member enterprises in the supply chain. At the same time, coordinated strategies can also improve the anti-epidemic efforts and the level of social welfare, which can alleviate the difficulties brought by the epidemic and effectively speed up the recovery of the whole society from the epidemic. The government should subsidize manufacturers who adopt a coordinated strategy, which can improve anti-epidemic efforts and social welfare. Management implications are not universal when the marginal benefits of anti-epidemic efforts are too small or too high.

On the other hand, the increase of the cost coefficient $\theta$ of anti-epidemic efforts will reduce the level of antiepidemic efforts and social welfare. In order to achieve a higher level of anti-epidemic efforts and social welfare, the government should introduce epidemic prevention and control policies, accelerate the process of epidemic normalization, accelerate the progress of enterprises' resumption of work and production, accelerate the research and development of vaccines, and accelerate the research and development of new and high technologies to improve logistics efficiency and reduce the cost coefficient of anti-epidemic efforts.

\subsection{Future research directions}

The first extension of the model proposed in this paper is that the anti-epidemic behavior of manufacturers can be quantified. In fact, when manufacturers are in the epidemic situation, their own production and transportation activities will improve the social economic level, accelerate the progress of social recovery from the epidemic, and will generate additional production costs. Secondly, the coordination strategies adopted by manufacturers and retailers may take various forms. This study mainly focuses on the cost-sharing strategy of anti-epidemic efforts, and subsequent studies can try to explore more coordination methods. Thirdly, this study focuses on the cooperation process initiated by retailers. The supply chain in reality will have a variety of driving modes, so we can try to compare and analyze the supply chain decisions driven by different subjects. Finally, the supply chain in reality is more diversified. In order to make our research more universal, subsequent studies can focus on the operation of the closed-loop supply chain or the supply chain with more participants in the epidemic situation.

\section{Appendix A. Derivation of Equilibrium}

\section{System $n N$}

We first analyze the benefit function of $R, R$ decides $e$ and $p$. Solving for the profit function of $R$ with respect to the second-order condition of $e$ and $p$, and we get Hessian Matrix $H^{R}(e, p)=\left[\begin{array}{ll}\frac{\partial^{2} \pi^{R}}{\partial e^{2}} & \frac{\partial^{2} \pi^{R}}{\partial e \partial p} \\ \frac{\partial^{2} \pi^{R}}{\partial p \partial e} & \frac{\partial^{2} \pi^{R}}{\partial p^{2}}\end{array}\right]=$ $\left[\begin{array}{ll}-\theta & 1 \\ 1 & -2\end{array}\right]$, when $2 \theta-1>0$ is satisfied, solving the first-order optimality condition for $e$ and $p$, i.e. $\frac{\partial \pi^{R}}{\partial e}=0$ 
and $\frac{\partial \pi^{R}}{\partial p}=0$, yields the optimal $e^{*}(\omega)=\frac{1-\omega}{2 \theta-1}$ and $p^{*}(\omega)=\frac{\theta \omega+\theta-\omega}{2 \theta-1}$. Then we analyze the profit function of $M$ to find the optimal $\omega$ by substituting $e^{*}(\omega)$ and $p^{*}(\omega)$ into benefit function of $M, \pi^{M}$ is concave in $\omega$ since $\frac{\partial^{2} \pi^{M}}{\partial \omega^{2}}=-\frac{2 \theta}{2 \theta-1}<0$, and solving $\frac{\partial \pi^{M}}{\partial \omega}=0$ gives: $\omega_{n N}=\frac{1+c}{2}$. Then, we get $p_{n N}=\frac{c \theta-c+3 \theta-1}{2(2 \theta-1)}, e_{n N}=\frac{1-c}{2(2 \theta-1)}$, $\omega_{n N}=\frac{1+c}{2}, q_{n N}=\frac{\theta(1-c)}{2(2 \theta-1)}, \pi_{n N}^{M}=\frac{\theta(1-c)^{2}}{4(2 \theta-1)}$ and $\pi_{n N}^{R}=\frac{\theta(1-c)^{2}}{8(2 \theta-1)}$.

\section{System $n Y$}

The first step is the same as above $n N$ case, we get Hessian Matrix $H^{R}(e, p)=\left[\begin{array}{ll}\frac{\partial^{2} \pi^{R}}{\partial e^{2}} & \frac{\partial^{2} \pi^{R}}{\partial e \partial p} \\ \frac{\partial^{2} \pi^{R}}{\partial p \partial e} & \frac{\partial^{2} \pi^{R}}{\partial p^{2}}\end{array}\right]=$ $\left[\begin{array}{ll}-\theta \lambda & 1 \\ 1 & -2\end{array}\right]$, when $2 \theta \lambda-1>0$ is satisfied, solving the first-order optimality condition for $e$ and $p$, i.e. $\frac{\partial \pi^{R}}{\partial e}=0$ and $\frac{\partial \pi^{R}}{\partial p}=0$, yields the optimal $e^{*}(\omega, \lambda)=\frac{1-\omega}{2 \lambda \theta-1}$ and $p^{*}(\omega, \lambda)=\frac{\lambda \theta \omega+\lambda \theta-\omega}{2 \lambda \theta-1}$. And then we analyze the profit function of $M$ to find the optimal $\omega$ and $\lambda$ by substituting $e^{*}(\omega, \lambda)$ and $p^{*}(\omega, \lambda)$ into benefit function of $M$, solving $\frac{\partial \pi^{M}}{\partial \omega}=0$ and $\frac{\partial \pi^{M}}{\partial \lambda}=0$ gives: $\lambda_{c Y}=\frac{2}{3}$ (the solution $\lambda=\frac{1}{2 \theta}$ is omitted, because it does not correspond to $2 \theta \lambda-1>0), \omega_{n Y}=\frac{8 c \theta-6 c+8 \theta-3}{16 \theta-9}$. Then, we get: $p_{n Y}=\frac{4 c \theta-6 c+12 \theta-3}{16 \theta-9}, e_{n Y}=\frac{6(1-c)}{16 \theta-9}, q_{n Y}=\frac{4 \theta(1-c)}{16 \theta-9}$, $\pi_{n Y}^{M}=\frac{2 \theta(1-c)^{2}}{16 \theta-9}$ and $\pi_{n Y}^{R}=\frac{4 \theta(1-c)(4 c \theta-3 c-4 \theta+3)}{16 \theta-9}$.

\section{System $c N$}

The first step is the same as above $n N$ case, we get Hessian Matrix $H^{R}(e, p)=\left[\begin{array}{ll}\frac{\partial^{2} \pi^{R}}{\partial e^{2}} & \frac{\partial^{2} \pi^{R}}{\partial e \partial p} \\ \frac{\partial^{2} \pi^{R}}{\partial p \partial e} & \frac{\partial^{2} \pi^{R}}{\partial p^{2}}\end{array}\right]=$ $\left[\begin{array}{ll}-\theta & 1 \\ 1 & -2\end{array}\right]$, when $2 \theta-1>0$ is satisfied, solving the first-order optimality condition for $e$ and $p$, i.e. $\frac{\partial \pi^{R}}{\partial e}=0$ and $\frac{\partial \pi^{R}}{\partial p}=0$, yields the optimal $e^{*}(\omega)=\frac{1-\omega}{2 \theta-1}$ and $p^{*}(\omega)=\frac{\theta \omega+\theta-\omega}{2 \theta-1}$. Then we analyze the profit function of $M$ to find the optimal $\omega$ by substituting $e^{*}(\omega)$ and $p^{*}(\omega)$ into benefit function of $M, \pi^{M}$ is concave in $\omega$ since $\frac{\partial^{2} \pi^{M}}{\partial \omega^{2}}=-\frac{2 \theta}{2 \theta-1}<0$, and solving $\frac{\partial \pi^{M}}{\partial \omega}=0$ gives: $\omega^{*}(s)=\frac{1+c-s}{2}, p^{*}(s)=\frac{c \theta-s \theta-c+s+3 \theta-1}{2(2 \theta-1)}$ and $e^{*}(s)=\frac{1-c+s}{2(2 \theta-1)}$. Lastly, substituting all of the above optimal decision variables into SW, SW is concave in $s$ when $\frac{\partial^{2} \mathrm{SW}}{\partial s^{2}}=\frac{-\theta(\theta-1)}{4(2 \theta-1)^{2}}<0$, i.e. $\theta-1>0$, solving $\frac{\partial \mathrm{SW}}{\partial s}=0$ give the optimal solutions:

$$
\begin{aligned}
& s_{c N}=\frac{-3 c \theta^{2}+4 \alpha \theta+c \theta+3 \theta^{2}-2 \alpha-\theta}{\theta(\theta-1)}, \quad \omega_{c N}=\frac{2 c \theta^{2}-2 \alpha \theta-c \theta-\theta^{2}+\alpha}{\theta(\theta-1)} \\
& e_{c N}=\frac{-c \theta+\alpha+\theta}{\theta(\theta-1)}, \quad p_{c N}=\frac{c \theta-\alpha}{\theta}, \quad q_{c N}=\frac{-c \theta+\alpha+\theta}{\theta-1}, \quad \pi_{c N}^{M}=\frac{(-c \theta+\alpha+\theta)^{2}(2 \theta-1)}{\theta(\theta-1)^{2}}, \\
& \pi_{c N}^{R}=\frac{(-c \theta+\alpha+\theta)^{2}(2 \theta-1)}{2 \theta(\theta-1)^{2}}, \quad \mathrm{SW}_{c N}=\frac{(-c \theta+\alpha+\theta)^{2}}{2 \theta(\theta-1)^{2}}
\end{aligned}
$$

\section{System $c Y$}

The first step is the same as above $n N$ case, we get Hessian Matrix $H^{R}(e, p)=\left[\begin{array}{ll}\frac{\partial^{2} \pi^{R}}{\partial e^{2}} & \frac{\partial^{2} \pi^{R}}{\partial e \partial p} \\ \frac{\partial^{2} \pi^{R}}{\partial p \partial e} & \frac{\partial^{2} \pi^{R}}{\partial p^{2}}\end{array}\right]=$ $\left[\begin{array}{ll}-\theta \lambda & 1 \\ 1 & -2\end{array}\right]$, when $2 \theta \lambda-1>0$ is satisfied, solving the first-order optimality condition for $e$ and $p$, i.e. $\frac{\partial \pi^{R}}{\partial e}=0$ and $\frac{\partial \pi^{R}}{\partial p}=0$, yields the optimal $e^{*}(\omega, \lambda)=\frac{1-\omega}{2 \lambda \theta-1}$ and $p^{*}(\omega, \lambda)=\frac{\lambda \theta \omega+\lambda \theta-\omega}{2 \lambda \theta-1}$. And then we analyze the profit function of $M$ to find the optimal $\omega$ and $\lambda$ by substituting $e^{*}(\omega, \lambda)$ and $p^{*}(\omega, \lambda)$ into benefit function of $M$, solving $\frac{\partial \pi^{M}}{\partial \omega}=0$ and $\frac{\partial \pi^{M}}{\partial \lambda}=0$ gives: $\lambda_{c Y}=\frac{2}{3}$ (the solution $\lambda=\frac{1}{2 \theta}$ is omitted, because it does not correspond to $2 \theta \lambda-1>0), \omega^{*}(s)=\frac{8 c \theta-8 s \theta-6 c+6 s+8 \theta-3}{16 \theta-9}, p^{*}(s)=\frac{4 c \theta-4 s \theta-6 c+6 s+12 \theta-3}{16 \theta-9}$, and $e^{*}(s)=\frac{6(1-c+s)}{16 \theta-9}$, note that the 
first-order condition is less than 0 and the second-order condition is greater than 0 when $16 \theta-9>0$. Lastly, substituting all of the above optimal decision variables into SW, SW is concave in $s$ when $\frac{\partial^{2} \mathrm{SW}}{\partial s^{2}}=\frac{-4 \theta(4 \theta-3)}{(16 \theta-9)^{2}}<0$, i.e. $4 \theta-3>0$, solving $\frac{\partial \mathrm{SW}}{\partial s}=0$ give the optimal solutions:

$$
\begin{aligned}
& s_{c Y}=\frac{3}{2} \frac{-8 c \theta^{2}+16 \alpha \theta+4 c \theta+8 \theta^{2}-9 \alpha-4 \theta}{\theta(4 \theta-3)}, \quad \omega_{c Y}=\frac{2 c \theta-3 \alpha-\theta}{\theta}, \\
& e_{c Y}=\frac{3(-2 c \theta+3 \alpha+2 \theta)}{\theta(4 \theta-3)}, \quad p_{c Y}=\frac{-4 c \theta^{2}+6 \alpha \theta+6 c \theta-9 \alpha-3 \theta}{\theta(4 \theta-3)}, \quad \lambda_{c Y}=\frac{2}{3}, \\
& q_{c Y}=\frac{2(-2 c \theta+3 \alpha+2 \theta)}{4 \theta-3}, \quad \pi_{c Y}^{M}=\frac{1}{2} \frac{(16 \theta-9)(-2 c \theta+3 \alpha+2 \theta)^{2}}{\theta(4 \theta-3)^{2}}, \\
& \pi_{c Y}^{R}=\frac{(-2 c \theta+3 \alpha+2 \theta)^{2}}{\theta(4 \theta-3)}, \quad \mathrm{SW}_{c Y}=\frac{1}{2} \frac{(-2 c \theta+3 \alpha+2 \theta)^{2}}{\theta(4 \theta-3)}
\end{aligned}
$$

\section{System $e N$}

The first step is the same as above $n N$ case, we get Hessian Matrix $H^{R}(e, p)=\left[\begin{array}{ll}\frac{\partial^{2} \pi^{R}}{\partial e^{2}} & \frac{\partial^{2} \pi^{R}}{\partial e \partial p} \\ \frac{\partial^{2} \pi^{R}}{\partial p \partial e} & \frac{\partial^{2} \pi^{R}}{\partial p^{2}}\end{array}\right]=$ $\left[\begin{array}{ll}-\theta & 1 \\ 1 & -2\end{array}\right]$, when $2 \theta-1>0$ is satisfied, solving the first-order optimality condition for $e$ and $p$, i.e. $\frac{\partial \pi^{R}}{\partial e}=0$ and $\frac{\partial \pi^{R}}{\partial p}=0$, yields the optimal $e^{*}(\omega, s)=\frac{1-\omega+2 s}{2 \theta-1}$ and $p^{*}(\omega, s)=\frac{\theta \omega+\theta-\omega+s}{2 \theta-1}$. Then we analyze the profit function of $M$ to find the optimal $\omega$ by substituting $e^{*}(\omega, s)$ and $p^{*}(\omega, s)$ into benefit function of $M, \pi^{M}$ is concave in $\omega$ since $\frac{\partial^{2} \pi^{M}}{\partial \omega^{2}}=-\frac{2 \theta}{2 \theta-1}<0$, and solving $\frac{\partial \pi^{M}}{\partial \omega}=0$ gives: $\omega^{*}(s)=\frac{c \theta+\theta+s}{2 \theta}, p^{*}(s)=\frac{c \theta^{2}-c \theta+3 \theta^{2}+3 \theta s-\theta-s}{2 \theta(2 \theta-1)}$ and $e^{*}(s)=\frac{c \theta-4 \theta s-\theta+s}{-2 \theta(2 \theta-1)}$. Lastly, substituting all of the above optimal decision variables into $\mathrm{SW}$, SW is concave in $s$ when $\frac{\partial^{2} \mathrm{SW}}{\partial s^{2}}=\frac{-16 \theta^{2}+16 \theta-3}{4 \theta(2 \theta-1)^{2}}<0$, solving $\frac{\partial \mathrm{SW}}{\partial s}=0$ give the optimal solutions:

$$
\begin{aligned}
s_{e N}= & \frac{16 \alpha \theta^{2}-3 c \theta^{2}-12 \alpha \theta+c \theta+3 \theta^{2}+2 \alpha-\theta}{16 \theta^{2}-15 \theta+3}, \\
\omega_{e N}= & \frac{8 c \theta^{3}+8 \alpha \theta^{2}-9 c \theta^{2}+8 \theta^{3}-6 \alpha \theta+2 c \theta-6 \theta^{2}+\alpha+\theta}{\theta\left(16 \theta^{2}-15 \theta+3\right)}, \\
e_{e N}= & \frac{16 \alpha \theta^{2}-7 c \theta^{2}-8 \alpha \theta+2 c \theta+7 \theta^{2}+\alpha-2 \theta}{\theta\left(16 \theta^{2}-15 \theta+3\right)}, \quad q_{e N}=\frac{(4 \theta-1)(-c \theta+\alpha+\theta)(2 \theta-1)}{32 \theta^{3}-46 \theta^{2}+21 \theta-3}, \\
p_{e N}= & \frac{(2 \theta-1)\left(4 c \theta^{3}+12 \alpha \theta^{2}-8 c \theta^{2}+12 \theta^{3}-7 \alpha \theta+2 c \theta-7 \theta^{2}+\alpha+\theta\right)}{\theta\left(32 \theta^{3}-46 \theta^{2}+21 \theta-3\right)}, \\
\pi_{e N}^{M}= & \frac{(4 \theta-1)^{2}(-c \theta+\alpha+\theta)^{2}(2 \theta-1)}{\theta\left(16 \theta^{2}-15 \theta+3\right)^{2}}, \\
\pi_{e N}^{R}= & \frac{32(c-1)^{2} \theta^{5}+\left(256 \alpha^{2}+160 \alpha(1-c)-23(c-1)^{2}\right) \theta^{4}+4(c+44 \alpha-1)(c-2 \alpha-1) \theta^{3}}{2 \theta\left(16 \theta^{2}-15 \theta+3\right)^{2}} \\
& \begin{aligned}
-144 c \theta^{4}+48 \alpha \theta^{3}+178 c \theta^{3}+144 \theta^{4}-56 \alpha \theta^{2}-67 c \theta^{2}-178 \theta^{3}+19 \alpha \theta \\
\left.+8 c \theta+67 \theta^{2}-2 \alpha-8 \theta\right)(-c \theta+\alpha+\theta)
\end{aligned} \\
\mathrm{SW}_{e N}= & \frac{2 \theta\left(16 \theta^{2}-15 \theta+3\right)^{2}}{7} .+19 \alpha^{2}
\end{aligned}
$$




\section{System eY}

The first step is the same as above $n N$ case, we get Hessian Matrix $H^{R}(e, p)=\left[\begin{array}{ll}\frac{\partial^{2} \pi^{R}}{\partial e^{2}} & \frac{\partial^{2} \pi^{R}}{\partial e \partial p} \\ \frac{\partial^{2} \pi^{R}}{\partial p \partial e} & \frac{\partial^{2} \pi^{R}}{\partial p^{2}}\end{array}\right]=$ $\left[\begin{array}{ll}-\theta \lambda & 1 \\ 1 & -2\end{array}\right]$, when $2 \theta \lambda-1>0$ is satisfied, solving the first-order optimality condition for $e$ and $p$, i.e. $\frac{\partial \pi^{R}}{\partial e}=0$ and $\frac{\partial \pi^{R}}{\partial p}=0$, yields the optimal $e^{*}(\omega, \lambda, s)=\frac{1-\omega+2 s}{2 \lambda \theta-1}$ and $p^{*}(\omega, \lambda, s)=\frac{\lambda \theta \omega+\lambda \theta-\omega+s}{2 \lambda \theta-1}$. And then we analyze the profit function of $M$ to find the optimal $\omega$ and $\lambda$ by substituting $e^{*}(\omega, \lambda, s)$ and $p^{*}(\omega, \lambda, s)$ into benefit function of $M$, solving $\frac{\partial \pi^{M}}{\partial \omega}=0$ and $\frac{\partial \pi^{M}}{\partial \lambda}=0$ gives: $\lambda^{*}(s)=\frac{2 c \theta-8 \theta s-2 \theta+3 s}{\theta(3 c-4 s-3)}$ (the solution $\lambda=\frac{1}{2 \theta}$ is omitted, because it does not correspond to $2 \theta \lambda-1>0), \omega^{*}(s)=\frac{8 c \theta-6 c+8 \theta+2 s-3}{16 \theta-9}, e^{*}(s)=\frac{-2(3 c-4 s-3)}{16 \theta-9}, p^{*}(s)=\frac{4 c \theta-6 c+12 \theta+5 s-3}{16 \theta-9}$. Lastly, substituting all of the above optimal decision variables into SW, SW is concave in $s$ when $\frac{\partial^{2} \mathrm{SW}}{\partial s^{2}}=-\frac{64 \theta-39}{(16 \theta-9)^{2}}<0$, i.e. $64 \theta-39>0$, solving $\frac{\partial \mathrm{SW}}{\partial s}=0$ give the optimal solutions:

$$
\begin{aligned}
s_{e Y} & =\frac{128 \alpha \theta-20 c \theta-72 \alpha+9 c+20 \theta-9}{64 \theta-39}, \quad \lambda_{e Y}=\frac{64 \alpha \theta-18 c \theta-24 \alpha+3 c+18 \theta-3}{\theta(32 \alpha-17 c+17)}, \\
\omega_{e Y} & =\frac{32 c \theta+16 \alpha-28 c+32 \theta-11}{64 \theta-39}, \quad e_{e Y}=\frac{2(32 \alpha-17 c+17)}{64 \theta-39}, \\
p_{e Y} & =\frac{16 c \theta+40 \alpha-31 c+48 \theta-8}{64 \theta-39}, \quad q_{e Y}=\frac{-16 c \theta+24 \alpha-3 c+16 \theta+3}{64 \theta-39}, \\
\pi_{e Y}^{M} & =\frac{512(c-1)^{2} \theta^{2}+\left(-46 c^{2}+(-2176 \alpha+92) c+2048 \alpha^{2}+2176 \alpha-46\right) \theta-135(c-8 \alpha-1)\left(c-\frac{16}{15} \alpha-1\right)}{(64 \theta-39)^{2}}, \\
\pi_{e Y}^{R} & =\frac{(4 \theta+5) c^{2}+c(-48 \alpha-8 \theta-10)+64 \alpha^{2}+48 \alpha+4 \theta+5}{64 \theta-39} \\
\mathrm{SW}_{e Y} & =\frac{(28 \theta+1) c^{2}+c(-68 \alpha-56 \theta-2)+64 \alpha^{2}+68 \alpha+28 \theta+1}{2(64 \theta-39)} .
\end{aligned}
$$

\section{System bN}

The first step is the same as above $n N$ case, we get Hessian Matrix $H^{R}(e, p)=\left[\begin{array}{ll}\frac{\partial^{2} \pi^{R}}{\partial e^{2}} & \frac{\partial^{2} \pi^{R}}{\partial e \partial p} \\ \frac{\partial^{2} \pi^{R}}{\partial p \partial e} & \frac{\partial^{2} \pi^{R}}{\partial p^{2}}\end{array}\right]=$ $\left[\begin{array}{ll}-\theta & 1 \\ 1 & -2\end{array}\right]$, when $2 \theta-1>0$ is satisfied, solving the first-order optimality condition for $e$ and $p$, i.e. $\frac{\partial \pi^{R}}{\partial e}=0$ and $\frac{\partial \pi^{R}}{\partial p}=0$, yields the optimal $e^{*}(\omega, s)=\frac{1-\omega+2 s}{2 \theta-1}$ and $p^{*}(\omega, s)=\frac{\theta \omega+\theta-\omega+s}{2 \theta-1}$. Then we analyze the profit function of $M$ to find the optimal $\omega$ by substituting $e^{*}(\omega, s)$ and $p^{*}(\omega, s)$ into benefit function of $M, \pi^{M}$ is concave in $\omega$ since $\frac{\partial^{2} \pi^{M}}{\partial \omega^{2}}=-\frac{2 \theta}{2 \theta-1}<0$, and solving $\frac{\partial \pi^{M}}{\partial \omega}=0$ gives: $\omega^{*}(s)=\frac{c \theta+\theta+s-s \theta}{2 \theta}, p^{*}(s)=\frac{c \theta^{2}-s \theta^{2}-c \theta+3 \theta^{2}+4 \theta s-\theta-s}{2 \theta(2 \theta-1)}$, and $e^{*}(s)=\frac{c \theta-5 \theta s-\theta+s}{-2 \theta(2 \theta-1)}$. Lastly, substituting all of the above optimal decision variables into $\mathrm{SW}, \mathrm{SW}$ is concave in $s$ when $\frac{\partial^{2} \mathrm{SW}}{\partial s^{2}}=\frac{\theta^{3}+17 \theta^{2}-17 \theta+3}{-4 \theta(2 \theta-1)^{2}}<0$, solving $\frac{\partial \mathrm{SW}}{\partial s}=0$ give the optimal solutions:

$$
\begin{aligned}
& s_{b N}=\frac{-3 c \theta^{3}+20 \alpha \theta^{2}-2 c \theta^{2}+3 \theta^{3}-14 \alpha \theta+c \theta+2 \theta^{2}+2 \alpha-\theta}{\theta^{3}+17 \theta^{2}-17 \theta+3}, \\
& \omega_{b N}=\frac{(2 c-1) \theta^{4}+(8 c-10 \alpha+9) \theta^{3}+(-10 c+17 \alpha-7) \theta^{2}+(2 c-8 \alpha+1) \theta+\alpha}{\theta\left(\theta^{3}+17 \theta^{2}-17 \theta+3\right)}, \\
& p_{b N}=\frac{c \theta^{4}+(2 c-5 \alpha+15) \theta^{3}+(-9 c+21 \alpha-8) \theta^{2}+(2 c-9 \alpha+1) \theta+\alpha}{\theta\left(\theta^{3}+17 \theta^{2}-17 \theta+3\right)}, \\
& e_{b N}=\frac{(-4 c+4) \theta^{3}+(-8 c+25 \alpha+8) \theta^{2}+(2 c-10 \alpha-2) \theta+\alpha}{\theta\left(\theta^{3}+17 \theta^{2}-17 \theta+3\right)},
\end{aligned}
$$




$$
\begin{aligned}
& q_{b N}=\frac{(-c+1) \theta^{3}+(-6 c+5 \alpha+6) \theta^{2}+(c+4 \alpha-1) \theta-\alpha}{\theta\left(\theta^{3}+17 \theta^{2}-17 \theta+3\right)}, \\
& \pi_{b N}^{M}=\frac{2\left((-c+1) \theta^{3}+(-6 c+5 \alpha+6) \theta^{2}+(-c-4 \alpha+1) \theta+\alpha\right)^{2}\left(\theta-\frac{1}{2}\right)}{\theta\left(\theta^{3}+17 \theta^{2}-17 \theta+3\right)^{2}}, \\
& 2(c-1)^{2} \theta^{7}+32(c-1)\left(c-1-\frac{5 \alpha}{8}\right) \theta^{6}+\left(50 \alpha^{2}+(-246 c+246) \alpha+68(c-1)^{2}\right) \theta^{5} \\
& +\left(455 \alpha^{2}-60(c-1)^{2}\right) \theta^{4}+\left(-588 \alpha^{2}+(160 c-160) \alpha+10(c-1)^{2}\right) \theta^{3}-60\left(c-1-\frac{127}{30} \alpha\right) \alpha \theta^{2} \\
& \pi_{b N}^{R}=\frac{+6 \alpha\left(c-1-\frac{23}{3} \alpha\right) \theta+3 \alpha^{2}}{\theta\left(\theta^{3}+17 \theta^{2}-17 \theta+3\right)^{2}}, \\
& \pi_{b N}^{G}=\frac{(c-1)^{2} \theta^{4}+9(c-1)\left(c-1-\frac{8}{9} \alpha\right) \theta^{3}+\left(25 \alpha^{2}+(-16 c+16) \alpha-2(c-1)^{2}\right) \theta^{2}+4\left(c-1-\frac{5}{2} \alpha\right) \alpha \theta+\alpha^{2}}{2 \theta\left(\theta^{3}+17 \theta^{2}-17 \theta+3\right)^{2}} .
\end{aligned}
$$

\section{Appendix B. Proof of propositions}

Proof of Proposition 4.1.

Part a

(i) $e_{c N}-e_{n N}=\frac{(1.5-1.5 c) \theta^{2}+(0.5 c-0.3) \theta-0.1}{\theta(\theta-1)(2 \theta-1)}>0$,

$$
\begin{aligned}
& e_{e N}-e_{n N}=\frac{(9.2-6 c) \theta^{3}+(3.5 c-6.7) \theta^{2}+(1.5-0.5 c) \theta-0.1}{\theta\left(16 \theta^{2}-15 \theta+3\right)(2 \theta-1)}>0, \\
& e_{b N}-e_{n N}=\frac{(7.5-7.5 c) \theta^{3}+(8.5-3.5 c) \theta^{3}+(3.5 c-8) \theta^{2}+(1.7-0.5 c) \theta-0.1}{\theta\left(\theta^{3}+17 \theta^{2}-17 \theta+3\right)(2 \theta-1)}>0 .
\end{aligned}
$$

(ii) $e_{c Y}-e_{n Y}=\frac{(72-72 c) \theta^{2}+(36 c-21.6) \theta-8.1}{\theta(4 \theta-3)(16 \theta-9)}>0, e_{e Y}-e_{n Y}=\frac{(1030.4-928 c) \theta-597.6+540 c}{\theta(64 \theta-39)(16 \theta-9)}>0$.

Part b

(i) $\mathrm{SW}_{c N}-\mathrm{SW}_{n N}=\frac{0.625(c-1)^{2} \theta^{3}+\left(-0.125 c^{2}+0.05 c+0.075\right) \theta^{2}+(0.1 c-0.09) \theta-0.005}{\theta(\theta-1)(2 \theta-1)}>0$,

$$
\begin{aligned}
& \mathrm{SW}_{e N}-\mathrm{SW}_{n N}=\frac{1}{\theta\left(16 \theta^{2}-15 \theta+3\right)^{2}(2 \theta-1)}\left(48(c-1)^{2} \theta^{6}+\left(-70 c^{2}+120.8 c-50.8\right) \theta^{5}+\left(35.625 c^{2}\right.\right. \\
& -38.25 c+3.105) \theta^{4}+\left(-7.75 c^{2}-4.8 c+11.75\right) \theta^{3}+\left(-0.625 c^{2}+4.05 c-4.205\right) \theta^{2} \\
& +(0.385-0.5 c) \theta-0.01<0 \text {, } \\
& 0.625(c-1)^{2} \theta^{5}+\left(2.125 c^{2}-5.05 c+2.925\right) \theta^{4}+\left(-0.125 c^{2}-0.95 c+1.325\right) \theta^{3} \\
& \mathrm{SW}_{b N}-\mathrm{SW}_{n N}=\frac{+\left(-0.125 c^{2}+1.45 c-1.55\right) \theta^{2}+(0.26-0.2 c) \theta-0.005}{\theta\left(\theta^{3}+17 \theta^{2}-17 \theta+3\right)^{2}(2 \theta-1)}>0 .
\end{aligned}
$$

(ii) $\mathrm{SW}_{c Y}-\mathrm{SW}_{n Y}=\frac{320(1-c)^{2} \theta^{4}+\left(-312 c^{2} 3+470.4 c-158.4\right) \theta^{3}+\left(72 c^{2}+28.8 c-89.28\right) \theta^{2}}{+(-48.6 c+35.64) \theta+3.645}>0$,

$$
\mathrm{SW}_{e Y}-\mathrm{SW}_{n Y}=\frac{512(1-c)^{2} \theta^{3}+\left(-112 c^{2}-646.4 c+840.32\right) \theta^{2}+\left(-180 c^{2}+1339.2 c-1251.36\right) \theta}{+40.5 c^{2}-356.4 c+341.82}>0 .
$$


Proof of Proposition 4.2.

Part a

(i) $\omega_{c N}=\frac{2 c \theta^{2}-2 \alpha \theta-c \theta-\theta^{2}+\alpha}{\theta(\theta-1)}>0$, i.e. $2(c-1) \theta^{2}-0.4 \theta-c \theta+0.2>0$, so

$$
e_{e N}-e_{b N}=\frac{(57 c-55.4) \theta^{5}-(49 c+324.6) \theta^{4}+(13 c+481.3) \theta^{3}-(c+219.9) \theta^{2}+40.9 \theta-2.7}{\theta\left(16 \theta^{2}-15 \theta+3\right)\left(\theta^{3}+17 \theta^{2}-17 \theta+3\right)}<0 .
$$

(ii) $e_{c N}-e_{e N}=\frac{(7.4-9 c) \theta^{3}+(6 c-2) \theta^{2}-(c+1.4) \theta+0.4}{\theta(\theta-1)\left(16 \theta^{2}-15 \theta+3\right)}$,

where $\psi_{e 1}=(7.4-9 c) \theta^{3}+\left(6 c-2 \theta^{2}-(c+1.4) \theta+0.4\right.$, so $e_{e N}<e_{c N}$, when $\psi_{e 1}>0$;

$e_{b N}-e_{c N}=\frac{(3 c-3) \theta^{4}-(-13 c+10.6) \theta^{3}-(7 c-1.8) \theta^{2}+(c+18) \theta-0.4}{\theta(\theta-1)\left(\theta^{3}+17 \theta^{2}-17 \theta+3\right)}$,

where $\psi_{e 2}=(3 c-3) \theta^{4}-(-13 c+10.6) \theta^{3}-(7 c-1.8) \theta^{2}+(c+18) \theta-0.4$, so $e_{b N}>e_{c N}$, when $\psi_{e 2}>0$.

Part b

(i) $e_{e N}-e_{e Y}=\frac{(96 c-96) \theta^{3}+(91.4-109 c) \theta^{2}+(24 c+5.6) \theta-3.9}{\theta(64 \theta-39)\left(16 \theta^{2}-15 \theta+3\right)}<0$,

$$
e_{c Y}-e_{e Y}=\frac{(222.4-248 c) \theta^{2}+(132 c-55.2) \theta-35.1}{\theta(4 \theta-3)(64 \theta-39)}>0 .
$$

(ii) $e_{c Y}-e_{c N}=\frac{\psi_{e 3}}{\theta(4 \theta-3)(\theta-1)}$,

where $\psi_{e 3}=(2-2 c) \theta^{2}-(2.5-3 c) \theta-0.6$, so $e_{c N}<e_{c Y}$, when $\psi_{e 3}>0$.

Part c

$$
\lambda_{e Y}-\lambda_{c Y}=\frac{128 \alpha \theta-20 c \theta-72 \alpha+9 c+20 \theta-9}{3 \theta(32 \alpha-17 c+17)}>0 .
$$

Proof of Proposition 4.3.

Part a

$$
\begin{aligned}
\mathrm{SW}_{e N}-\mathrm{SW}_{b Y}= & \frac{1}{\theta\left(16 \theta^{2}-15 \theta+3\right)^{2}\left(\theta^{3}+17 \theta^{2}-17 \theta+3\right)}\left(-56(c-1)^{2} \theta^{8}+\left(223 c^{2}-353.2 c+130.2\right) \theta^{7}\right. \\
& +\left(-448 c^{2}+757.3 c-341.06\right) \theta^{6}+\left(440.6+415 c^{2}-779 c^{2}\right) \theta^{5}+\left(-183 c^{2}+382.5 c-273.65\right) \theta^{4} \\
& \left.+\left(38 c^{2}-91.9 c+90.685\right) \theta^{3}+\left(-3 c^{2}+9.8 c-16.655\right) \theta^{2}+(1.655-0.3 c) \theta-0.075\right)<0 \\
\mathrm{SW}_{c N}-\mathrm{SW}_{b N}= & \frac{\left(4.2-8.7 c+4.5 c^{2}\right) \theta^{4}+\left(-1.82+4.7 c-3 c^{2}\right) \theta^{3}-\left(0.5 c^{2}-0.3 c+0.6\right) \theta^{2}+(0.5 c-0.4) \theta+0.02}{\theta(\theta-1)\left(\theta^{3}+17 \theta^{2}-17 \theta+3\right)^{2}} \\
> & 0 .
\end{aligned}
$$

Part b

(i) $\mathrm{SW}_{e Y}-\mathrm{SW}_{c Y}=\frac{\left(72 c^{2}-144 c+72\right) \theta^{3}-\left(-38 c^{2}+51.2 c-14.48\right) \theta^{2}+\left(-1.5 c^{2}+10.2 c-7.86\right) \theta-1.755}{\theta(4 \theta-3)(64 \theta-39)}<$ 0 . 
(ii) $\mathrm{SW}_{e Y}-\mathrm{SW}_{e N}=\frac{\psi_{\mathrm{SW} 1}}{\theta(64 \theta-39)\left(16 \theta^{2}-15 \theta+3\right)^{2}}$,

where $\psi_{\mathrm{SW} 1}=-(c-1)^{2} \theta^{6}-\left(-1912 c^{2}+4080 c-2249.92\right) \theta^{5}-\left(1361 c^{2}-3230.8 c+2038.76\right) \theta^{4}-\left(-463 c^{2}+\right.$ $1285.9 c-952.9) \theta^{3}-\left(75 c^{2}-256.3 c+227.1\right) \theta^{2}-\left(-4.5 c^{2}+20.1 c-22.825\right) \theta-0.39$.

Note that $0<c<1,1<\theta<3$, there are two solutions to $\psi_{\mathrm{SW} 1}=0$, but one of the solutions

$$
\begin{aligned}
c_{\mathrm{SW} 1_{1}}= & \frac{1}{\theta\left(2048 \theta^{5}-3824 \theta^{4}+2722 \theta^{3}-926 \theta^{2}+150 \theta-9\right)}\left(2048 \theta^{6}-4080 \theta^{5}+3230.8 \theta^{4}-1285.9 \theta^{3}+256.3 \theta^{2}\right. \\
& -20.1 \theta+\left(3355443.2 \theta^{11}-12530483.2 \theta^{10}+20101529.6 \theta^{9}-18102246.4 \theta^{8}+10044220.8 \theta^{7}\right. \\
& \left.\left.-3557299.1 \theta^{6}+813684 \theta^{5}-122943.9 \theta^{4}+13543.2 \theta^{3}-1238.4 \theta^{2}+70.2 \theta\right)^{\frac{1}{2}}\right)
\end{aligned}
$$

does not satisfy the condition, so

$$
\begin{aligned}
\mathrm{SW}_{e N}<\mathrm{SW}_{e Y} \Leftrightarrow & \psi_{\mathrm{SW} 1}>0 \Leftrightarrow c_{\mathrm{SW} 1_{2}}>-\frac{1}{\theta\left(2048 \theta^{5}-3824 \theta^{4}+2722 \theta^{3}-926 \theta^{2}+150 \theta-9\right)}\left(-2048 \theta^{6}+4080 \theta^{5}\right. \\
& -3230.8 \theta^{4}+1285.9 \theta^{3}-256.3 \theta^{2}+20.1 \theta+\left(3355443.2 \theta^{11}-12530483.2 \theta^{10}+20101529.6 \theta^{9}\right. \\
& -18102246.4 \theta^{8}+10044220.8 \theta^{7}-3557299.1 \theta^{6}+813684 \theta^{5}-122943.9 \theta^{4}+13543.2 \theta^{3} \\
& \left.\left.-1238.4 \theta^{2}+70.2 \theta\right)^{\frac{1}{2}}\right)
\end{aligned}
$$

$\mathrm{SW}_{c Y}-\mathrm{SW}_{c N}=\frac{\psi_{\mathrm{SW} 2}}{\theta(4 \theta-3)(\theta-1)}$,

where $\psi_{\mathrm{Sw} 2}=\left(-0.5 c^{2}+0.8 c-0.3\right) \theta^{2}+(0.3 c-0.275) \theta-0.03$. Note that $\omega_{c N}=\frac{2 c \theta^{2}-0.2 \theta-c \theta-\theta^{2}+0.1}{\theta(\theta-1)}, 0<c<$ $1,1<\theta<3$, there are two solutions to $\psi_{\mathrm{SW} 2}=0$, but one of the solutions $c_{\mathrm{SW} 2_{1}}=\frac{0.8 \theta+0.3+0.1 \sqrt{(\theta-1)(4 \theta-3)}}{\theta}$ does not satisfy the condition, so

$$
\mathrm{SW}_{c N}<\mathrm{SW}_{c Y} \Leftrightarrow \psi_{\mathrm{SW} 2}>0 \Leftrightarrow c>c_{\mathrm{SW}_{2}}=\frac{0.8 \theta+0.3-0.1 \sqrt{(\theta-1)(4 \theta-3)}}{\theta} .
$$

Proof of Proposition 4.4. $\frac{\partial e_{c N}}{\partial \theta}=\frac{(c-1) \theta^{2}+\alpha(1-2 \theta)}{\theta^{2}(\theta-1)^{2}}<0$; $\frac{\partial e_{C Y}}{\partial \theta}=\frac{24 \theta^{2}(c-1)+27 \alpha(1-3 \theta)}{\theta^{2}(4 \theta-3)}<0$; $\frac{\partial e_{e N}}{\partial \theta}(\alpha=0.1)=$ $\frac{(112 c-134) \theta^{4}+(-64 c+89.6) \theta^{3}+(9 c-21) \theta^{2}+3 \theta-0.3}{\theta^{2}\left(16 \theta^{2}-15 \theta+3\right)}$, and $(112 c-134) \theta^{4}+(-64 c+89.6) \theta^{3}+(9 c-21) \theta^{2}+3 \theta-0.3<$ $-22 \theta^{4}+89.6 \theta^{3}-12 \theta^{2}+3 \theta-0.3$, by deriving $-22 \theta^{4}+89.6 \theta^{3}-12 \theta^{2}+3 \theta-0.3$ many times, there is $-22 \theta^{4}+89.6 \theta^{3}-12 \theta^{2}+3 \theta-0.3<0$, i.e. $\frac{\partial e_{e N}}{\partial \theta}<0 ; \frac{\partial e_{e Y}}{\partial \theta}=\frac{-128(32 \alpha+17(1-c))}{(64 \theta-39)^{2}}<0 ; \quad \frac{\partial e_{b N}}{\partial \theta}(\alpha=0.1)=$ $\frac{(4 c-4) \theta^{6}+(16 c-21) \theta^{5}+(198 c-237.5) \theta^{4}+(-92 c+125.6) \theta^{3}+(10 c-24.6) \theta^{2}+3.4 \theta-0.3}{\theta^{2}\left(\theta^{3}+17 \theta^{2}-17 \theta+3\right)^{2}},(4 c-4) \theta^{6}+(16 c-21) \theta^{5}+(198 c-237.5) \theta^{4}+$ $(-92 c+125.6) \theta^{3}+(10 c-24.6) \theta^{2}+3.4 \theta-0.3<-5 \theta^{5}-39.5 \theta^{4}+125.6 \theta^{3}-14.6 \theta^{2}+3.4 \theta-0.3$, by deriving $-5 \theta^{5}-39.5 \theta^{4}+125.6 \theta^{3}-14.6 \theta^{2}+3.4 \theta-0.3$ many times, there is $-5 \theta^{5}-39.5 \theta^{4}+125.6 \theta^{3}-14.6 \theta^{2}+3.4 \theta-0.3<0$, i.e. $\frac{\partial e_{b N}}{\partial \theta}<0$.

Proof of Proposition 4.5.

$$
\begin{aligned}
\frac{\partial e_{e N}}{\partial \theta} & =\frac{-((c-2 \alpha-1) \theta+\alpha)((c-1) \theta-\alpha)}{2 \theta^{2}(\theta-1)^{2}}<0 ; \\
\frac{\partial \mathrm{SW}_{c Y}}{\partial \theta} & =-\frac{6\left((c-1) \theta-\frac{3}{2} \alpha\right)\left((c-1-4 \alpha) \theta+\frac{3}{2} \alpha\right)}{\theta^{2}(4 \theta-3)^{2}}<0 ; \\
\frac{\partial \mathrm{SW}_{e N}}{\partial \theta}(\alpha=0.1) & =\frac{1}{\theta^{2}\left(16 \theta^{2}-15 \theta+3\right)^{3}}\left(\left(-736 c^{2}+1625.6 c-889.6\right) \theta^{6}+\left(1127 c^{2}-2484.4 c+1349.72\right) \theta^{5}\right.
\end{aligned}
$$




$$
\begin{aligned}
& +\left(-609 c^{2}+1338 c-715.5\right) \theta^{4}+\left(141 c^{2}-308.3 c+158.46\right) \theta^{3}+\left(-12 c^{2}+26.1 c-11.29\right) \theta^{2} \\
& -0.45 \theta+0.3)<0 \\
\frac{\partial \mathrm{SW}_{e Y}}{\partial \theta}= & \frac{-2(-17 c+32 \alpha+17)^{2}}{(64 \theta-39)^{2}}<0 ; \\
\frac{\partial \mathrm{SW}_{b N}}{\partial \theta}(\alpha=0.1)= & \frac{1}{\theta^{2}\left(\theta^{3}+17 \theta^{2}-17 \theta+3\right)^{2}}\left(\left(4 c^{2}-7.6 c+3.6\right) \theta^{6}+\left(-15 c^{2}-16.85 c+31.6\right) \theta^{5}\right. \\
& +\left(-55 c^{2}+129.8 c-76.775\right) \theta^{4}+\left(27 c^{2}-63.2 c+37.88\right) \theta^{3}+\left(-3 c^{2}+7 c-4.73\right) \theta^{2} \\
& -0.17 \theta-0.015)<0 .
\end{aligned}
$$

Acknowledgements. This work is partially founded by the Humanities and Social Science Foundation of Ministry of Eduction (18YJA630088) and the Fundamental Research Funds for the Central Universities (2019JD2006).

\section{REFERENCES}

[1] M. Ando, C. Furukawa, D. Nakata and K. Sumiya, Fiscal responses to the covid-19 crisis in Japan: the first six months. Nat. Tax J. 73 (2020) 901-926.

[2] A. Arora and M. Ceccagnoli, Patent protection, complementary assets, and firms incentives for technology licensing. Manage. Sci. 52 (2006) 293-308.

[3] A. Atasu and R. Subramanian, Extended producer responsibility for e-waste: individual or collective producer responsibility? Prod. Oper. Manage. 21 (2012) 1042-1059.

[4] A. Atasu, L.N.V. Wassenhove and M. Sarvary, Efficient take-back legislation. Prod. Oper. Manage. 18 (2009) $243-258$.

[5] Y. Cao, Q.-S. Li and H. Hu, Research on the influence of different government subsidy strategies on the green decision-making of supply chain. Chin. J. Manage. 16 (2019) 297-305+316.

[6] D. Chakraborty, T. Garai, D.K. Jana, et al. A three-layer supply chain inventory model for non-instantaneous deteriorating item with inflation and delay in payments in random fuzzy environment[J]. J. Ind. Prod. Eng. 34 (2017) 407-424.

[7] J. Chen and P.C. Bell, Implementing market segmentation using full-refund and no-refund customer returns policies in a dual-channel supply chain structure. Int J. Prod. Econ. 136 (2012) 56-66.

[8] Z. Chen and S.-I.I. Su, Social welfare maximization with the least subsidy: photovoltaic supply chain equilibrium and coordination with fairness concern. Renew. Energy 132 (2019) 1332-1347.

[9] Z. Chen, S.-I.I. Su and H. Wang, Inter-basin water transfer supply chain equilibrium and coordination: a social welfare maximization perspective. Water Res. Manage. 33 (2019) 2577-2598.

[10] J.-Y. Chen, S. Dimitrov and H. Pun, The impact of government subsidy on supply chains' sustainability innovation. Omega-Int. J. Manage. Sci. 86 (2019) 42-58.

[11] X. Chen, K. Li, F. Wang and X. Li, Optimal production, pricing and government subsidy policies for a closed loop supply chain with uncertain returns. J. Ind. Manage. Optim. 13 (2020) 1.

[12] T.-M. Choi, Innovative Bring-Service-Near-Your-Home Operations under Corona-Virus (COVID-19/SARS-CoV-2) outbreak: can logistics become the messiah? Transp. Res. Part E-Logistics Transp. Rev. 140 (2020) 101961.

[13] J. Fan, D. Ni and X. Fang, Liability cost sharing, product quality choice, and coordination in two-echelon supply chains. Eur. J. Oper. Res. 284 (2020) 514-537.

[14] M. Ferguson, V.D. Guide, G.C. Souza, et al. Supply chain coordination for false failure returns. Manuf. Serv. Oper. Manag. 8 (2006) 376-393.

[15] T. Garai and H. Garg, Multi-objective linear fractional inventory model with possibility and necessity constraints under generalised intuitionistic fuzzy set environment[J]. CAAI Trans. Intell. Technol. 4 (2019) P175-P181.

[16] T. Garai, D. Chakraborty and T.K. Roy, Fully fuzzy inventory model with price-dependent demand and time varying holding cost under fuzzy decision variables[J]. J. Intell. Fuzzy Syst. 36 (2019) 3725-3738.

[17] D. Ghosh and J. Shah, Supply chain analysis under green sensitive consumer demand and cost sharing contract. Int. J. Prod. Econ. 164 (2015) 319-329.

[18] K. Govindan, H. Mina and B. Alavi, A decision support system for demand management in healthcare supply chains considering the epidemic outbreaks: a case study of coronavirus disease 2019 (COVID-19). Transp. Res. Part E-Logistics Transp. Rev. 138 (2020) 101967.

[19] Q. Han, Y. Wang, L. Shen and W. Dong, Decision and coordination of low-carbon e-commerce supply chain with government carbon subsidies and fairness concerns. Complexity 2020 (2020) 1-19.

[20] I.-H. Hong, P.-C. Chen and H.-T. Yu, The effects of government subsidies on decentralised reverse supply chains. Int. J. Prod. Res. 54 (2016) 3962-3977. 
[21] S. Huang, Z.-P. Fan and X. Wang, Optimal operational strategies of supply chain under financing service by a 3PL firm. Int. J. Prod. Res. 57 (2019) 3405-3420.

[22] M. Jian, F. Sun and S. Hayrutdinov, Analysis of government differential weight subsidy in an emerging coastal industry supply chain. J. Coastal Res. 94 (2019) 851-858.

[23] Z.-Z. Jiang, N. He, L. Xiao and Y. Sheng, Government subsidy provision in biomass energy supply chains. Enterprise Inf. Syst. 13 (2019) 1367-1391.

[24] C.L. Karmaker, T. Ahmed, S. Ahmed, S.M. Ali, A. Moktadir and G. Kabir, Improving supply chain sustainability in the context of COVID-19 pandemic in an emerging economy: exploring drivers using an integrated model. Sustainable Prod. Consump. $\mathbf{2 6}$ (2021) 411-427.

[25] S. Kuo, H.-T. Ou and C.J. Wang, Managing medication supply chains: lessons learned from Taiwan during the COVID-19 pandemic and preparedness planning for the future. J. Am. Pharmacists Assoc. 61 (2021): e12-e15.

[26] Y. Li, X. Xie and Q. Liu, Study on longitudinal emission reduction investment of supply chain and government's subsidy policy. J. Intell. Fuzzy Syst. 34 (2018) 1177-1186.

[27] X. Li, H. Kuang and Y. Hu, Using system dynamics and game model to estimate optimal subsidy in shore power technology. IEEE Access 8 (2020) 116310-116320.

[28] Y. Li, K. Chen, S. Collignon and D. Ivanov, Ripple effect in the supply chain network: forward and backward disruption propagation, network health and firm vulnerability. Eur. J. Oper. Res. 291 (2021) 1117-1131.

[29] D. Loske, The impact of COVID-19 on transport volume and freight capacity dynamics: an empirical analysis in German food retail logistics. Transp. Res. Interdisciplinary Perspect. 6 (2020) 100165.

[30] W. Ma, Z. Zhao and H. Ke, Dual-channel closed-loop supply chain with government consumption-subsidy. Eur. J. Oper. Res. 226 (2013) 221-227.

[31] A. Majumdar, M. Shaw and S.K. Sinha, COVID-19 debunks the myth of socially sustainable supply chain: a case of the clothing industry in South Asian Countries. Sustainable Prod. Consump. 24 (2020) 150-155.

[32] Q. Meng, M. Li, Z. Li and J. Zhu, How different government subsidy objects impact on green supply chain decision considering consumer group complexity. Math. Prob. Eng. 2020 (2020) 1-12.

[33] Ministry of Power. National Ujala Dashboard |EESL Retrieved November 19, 2017, from http://www.ujala.gov.in/.

[34] Municipal notice on novel Coronavirus Infected pneumonia Epidemic Prevention and control Headquarters. Retrieved from http://www.wh.gov.cn/sy/whyw/202003/t20200316_960171.shtml.

[35] C.J.C. Nicomedes and R.M.A. Avila, An analysis on the panic during COVID-19 pandemic through an online form. J. Affective Disorders 276 (2020) 14-22.

[36] M. Pu and Y. Zhong, Rising concerns over agricultural production as COVID-19 spreads: lessons from China. Global Food Secur. 26 (2020) 100409.

[37] G. Raz and A. Ovchinnikov, Coordinating pricing and supply of public interest goods using government rebates and subsidies. IEEE Trans. Eng. Manage. 62 (2015) 65-79.

[38] Real-time big data reporting on COVID-19. Retrieved from https://voice.baidu.com/act/newpneumonia/newpneumonia/ ?from=osari_pc_3.

[39] M. Rizou, I.M. Galanakis, T.M.S. Aldawoud and C.M. Galanakis, Safety of foods, food supply chain and environment within the COVID-19 pandemic. Trends Food Sci. Technol. 102 (2020) 293-299.

[40] N.J. Rowan and J.G. Laffey, Challenges and solutions for addressing critical shortage of supply chain for personal and protective equipment (PPE) arising from Coronavirus disease (COVID19) pandemic - Case study from the Republic of Ireland. Sci. Total Environ. 725 (2020) 138532.

[41] A. Sharma, A. Adhikary and S.B. Borah, Covid-19's impact on supply chain decisions: strategic insights from NASDAQ 100 firms using Twitter data. J. Bus. Res. 117 (2020) 443-449.

[42] C. Su, X. Liu and W. Du, Green supply chain decisions considering consumers' low-carbon awareness under different government subsidies. Sustainability 12 (2020) 2281.

[43] H. Sun, Y. Wan, L. Zhang and Z. Zhou, Evolutionary game of the green investment in a two-echelon supply chain under a government subsidy mechanism. J. Cleaner Prod. 235 (2019) 1315-1326.

[44] Z. Wang, J. Huo and Y. Duan, Impact of government subsidies on pricing strategies in reverse supply chains of waste electrical and electronic equipment. Waste Manage. 95 (2019) 440-449.

[45] L. You-don, Governmental subsidy policy for enterprises' cooperative behavior in emission reduction in low-carbon supply chain. Oper. Res. Manage. Sci. 23 (2014) 1-11. 